\title{
Guías Latinoamericanas de Hipertensión Arterial
}

\author{
Ramiro A. Sánchez, Miryam Ayala, Hugo Baglivo, Carlos Velázquez, Guillermo Burlando, \\ Oswaldo Kohlmann, Jorge Jiménez, Patricio López Jaramillo, Ayrton Brandao, Gloria \\ Valdés, Luis Alcocer, Mario Bendersky, Agustín José Ramírez, Alberto Zanchetti, de parte \\ del Grupo Latinoamericano de Expertos.
}

Recibido el 15 de Diciembre de 2009, Aceptado el 2 de Marzo de 2010

Rev Chil Cardiol 2010; 29: 117-144

La hipertensión es un factor de riesgo cardiovascular muy prevalente en el mundo, y especialmente abrumador en los países de bajos y medianos ingresos. Informes recientes de la OMS y del Banco Mundial destacan la importancia de las enfermedades crónicas tales como la hipertensión, como obstáculo al logro de un buen estado de salud. Se debe agregar que, para la mayoría de los países de bajos y medianos ingresos, estrategias deficientes de la atención primaria de la salud son obstáculos mayores par el logro del control de la presión arterial. Es más, la epidemiología de la hipertensión y enfermedades relacionadas, los recursos y las prioridades de salud, el estado socioeconómico de la población, varían considerablemente en diferentes países y en diferentes regiones de países individuales. Teniendo en cuenta las bajas tasas de control de la presión arterial logrados en Latinoamérica y los beneficios que se puede esperar de un mejor control, se decidió invitar a especialistas de diferentes países latinoamericanos a analizar la situación de la región y redactar un documento de consenso sobre la detección, evaluación y tratamiento de la hipertensión que podría ser adecuado del punto de vista costo-utilidad. Las recomendaciones incluidas aquí son el resultado de documentos preparatorios escritos por expertos invitados y el muy activo debate posterior en diferentes paneles de discusión, realizados durante dos días en Asunción, Paraguay en Mayo del año 2008. Por último, para mejorar la práctica clínica, la publicación de estas pautas debe ser seguida por la implementación de intervenciones efectivas capaces de vencer las barreras (cognitivas, de comportamiento y afectivas) que previenen los cambios de actitud tanto en médicos como en pacientes.

Nota: Estas guías han sido publicadas como Latin American guidelines on hypertension. Latin American Expert Group. J Hypertens 2009; 27:905-922.

Correspondencia: Dr. Ramiro Sánchez

Sección Hipertensión Arterial y Unidad Metabólica

Fundación Favaloro

Belgrano 1782 P. 4 - Buenos Aires - Argentina

Correo Electrónico: rsanchez@ffavaloro.org 


\section{Introducción}

La hipertensión es un factor de riesgo cardiovascular muy prevalente en el mundo, que es especialmente abrumador en los países de bajos y medianos ingresos. Informes recientes de la OMS'y del Banco Mundial ${ }^{2}$ destacan la importancia de las enfermedades crónicas tales como la hipertensión, como obstáculo al logro de un buen estado de salud. Se debe agregar que, para la mayoría de los países de bajos y medianos ingresos, estrategias deficientes de la atención primaria de la salud son obstáculos mayores par el logro del control de la presión arterial ${ }^{3}$. Es más, la epidemiología de la hipertensión y enfermedades relacionadas, los recursos y las prioridades de salud, el estado socioeconómico de la población, varían considerablemente en diferentes países y en diferentes regiones de países individuales. A causa de esto, los documentos de la Organización Mundial de la Salud - Sociedad Internacional de Hipertensión ${ }^{4}$ y de la Sociedad Europea de Hipertensión - Sociedad Europea de Cardiología ${ }^{5}$ recomiendan el desarrollo de pautas locales que tengan en cuenta estas condiciones.

Teniendo en cuenta las bajas tasas de control de la presión arterial logrados en Latino América y los beneficios que se puede esperar de un mejor control, se decidió invitar a especialistas de diferentes países latinoamericanos a analizar la situación de la región y redactar un documento de consenso sobre la detección, evaluación y tratamiento de la hipertensión que podría ser adecuado del punto de vista costo-utilidad. Es por eso que miembros de las Sociedades de Hipertensión, Cardiología y Diabetes de países latinoamericanos se reunieron para elaborar las nuevas recomendaciones para la prevención y el manejo de la hipertensión y enfermedades relacionadas, y redactar un documento de consenso, con atención especial al Síndrome Metabólico (SM) para alertar a los médicos acerca de esta condición de mayor riesgo, especialmente prominente en Latinoamérica pero habitualmente subestimada y subtratada. El documento resultante está diseñado para servir como guía a los médicos que atienden pacientes con hipertensión y comorbilidades.

Las recomendaciones incluidas aquí son el resultado de documentos preparatorios escritos por expertos invitados y el siguiente debate muy activo por diferentes paneles de discusión, realizados en sesiones de 2 días en Asunción, Paraguay en mayo del año 2008. Después de una presentación formal de las conclusiones finales de cada panel de discusión y su aprobación por todos los participantes, este documento final fue preparado por un Comité de Redacción designado. Para mejorar la práctica clínica, la publicación de estas pautas debe ser seguida por la implementación de intervenciones efectivas capaces de vencer las barreras (cognitivas, de comportamiento y afectivas) que previenen los cambios de actitud tanto en médicos como en pacientes.

\section{Epidemiología - Economía de Salud - Educación - Prevalencia}

La diabetes mellitus y la hipertensión frecuentemente están asociadas, aumentando así su efecto negativo sobre el sistema cardiovascular ${ }^{6,7}$. Más del $80 \%$ de la carga mundial atribuida a estas enfermedades son en países de bajos y medianos ingresos. En Latinoamérica, el $13 \%$ de las muertes y el $5,1 \%$ de los años de vida ajustados por discapacidad (AVAD) pueden ser atribuidos a la hipertensión ${ }^{1}$. La prevalencia ajustada para la edad de la hipertensión en la población adulta general en diferentes países de Latinoamérica (encuestas nacionales o muestreos sistemáticos aleatorizados) varía entre el 26 al $42 \%$-9. En las poblaciones diabéticas, la prevalencia de la hipertensión es 1,5 a 3 veces mayor que en no diabéticos de la misma franja etaria. En la diabetes tipo 2, la hipertensión puede ya estar presente en el momento del diagnóstico o inclusive puede preceder a la hiperglicemia evidente ${ }^{6}$.

Las tablas 1 y 2 muestran la prevalencia, el grado de detección, el tratamiento y el control de la hipertensión, junto con la mortalidad cardiovascular (CV) atribuida a la hipertensión, y a la prevalencia de los principales factores de riesgo asociados con la hipertensión respectivamente, en varios países latinoamericanos. 
Tabla1: Hipertensión Arterial, Sexo y mortalidad CV

\begin{tabular}{lccccccc}
\hline Paises & $\begin{array}{c}\text { Prevalencia } \\
\text { Hipertensión } \\
\%\end{array}$ & $\begin{array}{c}\text { Conciencia } \\
\text { Hipertensión } \\
\%\end{array}$ & $\begin{array}{c}\text { Hipertensión } \\
\text { Tratad } \\
\%\end{array}$ & $\begin{array}{c}\text { Hipertensión } \\
\text { Controlada } \\
\%\end{array}$ & $\%$ & $\begin{array}{c}\text { \% } \\
\text { Mortalidad } \\
\text { CV } \\
\%\end{array}$ \\
\hline Argentina & 28,1 & 54 & 42 & 18 & - & - & 23,5 \\
Brasil & $25-35$ & 50,8 & 40,5 & 10,2 & & & 27,5 \\
Chile & 33,7 & 59,8 & 36,3 & 11,8 & 30,8 & 36,7 & 28,4 \\
Colombia & 23 & 41 & 46 & 15 & & & 28 \\
Ecuador & 28,7 & 41 & 23 & 6,7 & 27,5 & 30,9 & 28 \\
México & 30,8 & 56,4 & 23 & 19,2 & 26,3 & 34,2 & - \\
Paraguay & 35 & 31 & 27 & 7 & & & 28 \\
Perú & 24 & 39 & 14,7 & 14 & & & - \\
Uruguay & 33 & 68 & 48 & 11 & 56,9 & 43,1 & 29,5 \\
Venezuela & 33 & 55 & 30 & 12 & & & 20,6 \\
\hline
\end{tabular}

Las columnas 2, 3 y 4 muestran valores de porcentages que corresponden a la población hipertensa correspondiente (columna 1).

Tabla2: Prevalencia de factores de Riesgo Asociados con HTA

\begin{tabular}{lcccc}
\hline & $\begin{array}{c}\text { Sobrepeso } \\
\%\end{array}$ & $\begin{array}{c}\text { Sedentarismo } \\
\%\end{array}$ & $\begin{array}{c}\text { Tabaquismo } \\
\%\end{array}$ & $\begin{array}{c}\text { Dislipidemia } \\
\%\end{array}$ \\
\hline Argentina & 19,7 & $\mathrm{Nd}$ & 38,6 & 18,7 \\
Brasil & 13 & $\mathrm{Nd}$ & 20 & 13 \\
Chile & 23,2 & 90,8 & 42 & 35,4 \\
Colombia & 47 & 61 & 23 & 61 \\
Ecuador & 41 & 34,9 & 24,8 & - \\
México & 31 & 30,8 & 36,6 & 36,5 \\
Paraguay & 54 & 38 & 34 & - \\
Perú & - & - & - & 10 \\
Uruguay & 59,7 & 64,3 & 15,7 & 18 \\
Venezuela & 25,1 & - & 30 & 5,7 \\
\hline
\end{tabular}

\section{Economía médica}

La hipertensión impone una enorme carga económica y social mundial a causa de las comorbilidades asociadas y de las complicaciones crónicas que pueden afectar la sobrevida y la calidad de vida. Así, un análisis reciente de un banco de datos internacional ${ }^{1}$ ha demostrado que una proporción muy substancial de la enfermedad cardiovascular es atribuible a la hipertensión.

Los gastos globales en el tratamiento antihipertensivo son de unos 50 mil millones de dólares anuales ${ }^{10}$. Más del $90 \%$ de los cuales se gastan en países de altos ingresos, mientras que los países de bajos y medianos ingresos, a pesar de tener una carga de enfermedad más de cinco veces mayor que los correspondientes países de altos ingresos, tienen acceso a solo el $10 \%$ de los recursos globales de tratamiento. Los parámetros de costo-efectividad, costo-beneficio y costo-utilidad del tratamiento de la hipertensión en la población general son muy afectados por la presencia de comorbilidades y complicaciones ${ }^{11-13}$. Teniendo en cuenta los datos antedichos, cabe esperar que la propuesta de un 
tratamiento intensivo de la hipertensión disminuya los costos y mejore la sobrevida y la calidad de vida.

\section{Educación}

En el contexto de una gran y creciente carga de enfermedad, las estrategias para mejorar la salud de la población necesitan un manejo consistente e integral de los principales factores de riesgo que contribuyen a la incapacidad y mortalidad prematura. La educación al personal de salud se debe considerar una herramienta importante para mejorar las estrategias de tratamiento de la hipertensión. Con este fin, las recientemente publicadas Pautas de la OMS/ISH sobre la cardiología preventiva ayudan en gran medida a los médicos generalistas en el manejo de estrategias globales de riesgo, con pautas de bolsillo que se puede obtener fácilmente en diferentes idiomas: (https://www.who.int./cardiovascular_diseases/ guidelines/Pocket_GL_information/en/index.html). Además, se recomienda enfáticamente la educación sistemática de los pacientes hipertensos. Este esfuerzo de educación debe involucrar a los pacientes en su propio tratamiento, y ayudar a la formación de los equipos de salud en el área de educación de pacientes. Se ha propuesto algunos programas educacionales para Latinoamérica ${ }^{14}$. La promoción de la educación en la población general, y sobre todo en sujetos de alto riesgo, es muy aconsejable. La educación formal para niños y adolescentes debe incluir información acerca de estilos de vida sanos.

Se recomienda las siguientes estrategias:

1 Programas comunitarios de educación

2 Estrategias operacionales para promover cambios de estilo de vida sobre todo en niños, adolescentes y adultos jóvenes.

3 Programas educacionales para médicos y equipos de salud (enfermeras, nutricionistas, etc.)

4 Programas de detección precoz de la hipertensión, y

5 Pautas para el control óptimo de las cifras tensionales.

\section{Características Clínicas}

Definición:

La hipertensión establecida es una condición que implica un mayor riesgo de eventos cardiovasculares y disminución de la función de diferentes órganos en la cual la presión arterial está crónicamente elevada por encima de valores considerados óptimos o normales. La hipertensión frecuentemente está asociada con comorbilidades tales como diabetes mellitus, coronariopatía, insuficiencia cardiaca (IC) crónica, accidente vascular encefálico (AVE), accidente isquémico transitorio (AIT), enfermedad vascular periférica, insuficiencia renal crónica. La hipertensión persistente es considerada uno de los factores de riesgo para $A V E$, infarto de miocardio, IC y aneurisma arterial, y es una de las principales causas de insuficiencia renal crónica y diálisis. Incluso una moderada elevación de la presión arterial conlleva una disminución de la expectativa de vida. Cuando la presión arterial está marcadamente elevada (presión arterial media 50\% o más por encima del promedio) la expectativa de vida se disminuye en 30 a $40 \%$, a menos que la hipertensión sea tratada adecuadamente ${ }^{15}$.

\section{Clasificación de Presión Arterial:}

Luego de considerar las clasificaciones propuestas por el Séptimo Informe del Comité Nacional Conjunto sobre la Prevención, Detección, Evaluación y Tratamiento de la Presión Arterial Elevada ${ }^{16}$ las Pautas Europeas para el Manejo de la Hipertensión $2007^{5}$, y el anterior Consenso Latinoamericano sobre Hipertensión Arterial ${ }^{17}$, se decidió, como se muestra en el Cuadro 3, mantener el concepto de que la hipertensión se diagnostica cuando la presión arterial es $\geq 140 / 90 \mathrm{mmHg}$. Por encima de este valor, se puede dividir a la hipertensión en grado 1, 2 o 3 . Esta clasificación también se aplica a la hipertensión sistólica aislada, que debe ser diagnosticada y tratada sobre todo en los pacientes mayores. 
Tabla 3: Clasificación de Presión Arterial

\begin{tabular}{|lll|}
\multicolumn{2}{c}{ Presión Arterial } \\
\hline Optima & & $<120 / 80 \mathrm{mmHg}$ \\
\hline Normal: & & $120 / 80-129 / 84 \mathrm{mmHg}$ \\
Normal Alta: & & $130 / 85-139 / 89 \mathrm{mmHg}$ \\
\hline Hipertensión & Grado 1: & $140-159 / 90-99 \mathrm{mmHg}$ \\
Hipertensión & Grado 2: & $160-179 / 100-109 \mathrm{mmHg}$ \\
Hipertensión & Grado 3: & $\geq 180 / 110 \mathrm{mmHg}$ \\
\hline Hipertensión Sistólica Aislada & $\geq 140 /<90 \mathrm{mmHg}$ \\
\hline
\end{tabular}

Teniendo en cuenta que la presión arterial es una variable continua, y que a mayores cifras tensionales mayor es el riesgo cardiovascular ${ }^{18,19}$, se decidió que los sujetos con PA entre 120/80 y 129/84 pueden ser considerados presión normal, mientras que los que tienen cifras entre 130/85 y 139/89 son considerados presión arterial normal alta. Los valores de presión arterial menores de 120/80 son considerados valores óptimos. Se debe enfatizar que los valores normales altos y normales son de mayor riesgo que los valores óptimos, a pesar de estar en el rango normal. La hipertensión arterial generalmente se clasifica como: Primaria, Esencial o Idiopática cuando la presión arterial en constantemente mayor de lo normal, sin causa subyacente conocida. Representa el 85 a 90\% de todos los casos de hipertensión. La hipertensión es definida como Secundaria cuando la presión arterial es elevada como resultado de una causa subyacente identificable, frecuentemente corregible (el restante 10 a $15 \%$ de los sujetos hipertensos).

\section{La Hipertensión Resistente o Refractaria al Trata-} miento representa una elevación de presión arterial que se mantiene por encima de los valores fijados como objetivo a pesar de la institución de tratamiento no farmacológico y farmacológico incluyendo dosis plenas de tres o más medicamentos, uno de los cuales un diurético. Estos pacientes deben ser remitidos a un especialista o a un centro de hipertensión porque este tipo de hipertensión frecuentemente está asociado con daño subclínico de órganos blanco, y tiene un mayor riesgo cardiovascular ${ }^{20}$.

Hipertensión de Bata Blanca: también llamada Hipertensión Aislada del Consultorio, es la condición en la cual la presión arterial medida en el consultorio está constantemente en el rango hipertenso, mientras que los valores medios de MAPA $^{21}$ o domiciliarios ${ }^{22}$ siempre están en rango normotenso. Su prevalencia es de alrededor del $10 \%$. Su riesgo general no está claramente establecido ${ }^{23}$ pero parece estar asociada con más anormalidades cardiacas, renales y metabólicas funcionales y/o estructurales que la plena normotensión ${ }^{24,25}$.

Hipertensión Oculta o Enmascarada: También llamada Hipertensión Ambulatoria Aislada, representa la condición contraria a la hipertensión de bata blanca, es decir, los sujetos tienen cifras tensionales normalesenelconsultoriomientrasquelosvaloresmedios de monitoreo ambulatorio de presión arterial (MAPA) o los valores domiciliarios están en rango hipertenso. Se encuentra en uno de cada 7 a 8 sujetos con valores normales en el consultorio ${ }^{25}$. El riesgo cardiovascular en estos pacientes parece ser similar al de los hipertensos establecidos ${ }^{26,27}$. Por ende, se debe tener 
R. Sánchez, M. Ayala, H. Baglivo, C. Velázquez, G. Burlando, O. kohlmann, et. al.

cuidado para evitar que estos sujetos vayan sin ser diagnosticados, usando MAPA o mediciones domiciliarias de la PA.

Hipertensión Sistólica Aislada: Es la presión arterial sistólica (PAS) constantemente $\geq 140 \mathrm{~mm} \mathrm{Hg}$ con presión arterial diastólica (PAD) $>90 \mathrm{~mm} \mathrm{Hg}$. Como la PAS tiende a subir con la edad, la prevalencia de la hipertensión sistólica aumenta con la edad, y por encima de los 60 años de edad representa una forma común de hipertensión. Se ha acumulado sólida evidencia acerca de la importancia de la PAS como factor de riesgo mayor para las enfermedades cardiovasculares ${ }^{28}$.

\section{Estratificación de Riesgos}

Para manejar un paciente hipertenso se debe tener en cuenta no solo los niveles tensionales, sino el riesgo cardiovascular total. Para estratificar el riesgo cardiovascular total, se debe tener en cuenta una serie de factores de riesgo, la presencia de daño de órganos blanco, y de otras condiciones o resultados clínicos previos o concomitantes (Tabla 4) asociados con la presión arterial, como se muestra en la Figura 1.

Entre los factores de riesgo tradicionales, las condiciones socio-económicas deben recibir atención especial en Latinoamérica. Del mismo modo se debe hacer énfasis en el bajo nivel de educación, a causa del alto porcentaje de población nativa con bajas oportunidades de obtener una educación adecuada.

La Figura 1 no solo incluye los valores tradicionales de corte de 140/90 mmHg, sino también los que se consideran óptimos o normales, o normales altos. A todos los niveles de presión arterial, incluyendo los óptimos, el riesgo total aumenta progresivamente al ir agregándose otros factores de riesgo, daño de órganos blanco, diabetes y resultados previos. Las influencias de la estratificación total de riesgos sobre las decisiones terapéuticas se describen en la sección sobre Selección de drogas antihipertensivas.

Tabla 4: Factores que tener en cuenta para cuantificar el riesgo cardiovascular

\begin{tabular}{|c|c|c|}
\hline Facores de Riesgo & Subclínicos & Eventos Clínicos \\
\hline $\begin{array}{l}\text { - Edad, Sexo (Masculino), } \\
\text { - Hipertensión, } \\
\text { - Colesterol Total, } \\
\text { - Tabaquismo, Diabetes*, } \\
\text { - Anteced. familiar de Eventos CV, } \\
\text { - HDLcolbajo, } \\
\text { - HDLcolalto, } \\
\text { - Sobrepesp/Obesidad } \\
\text { (IMC>25Kg/m²), } \\
\text { - Menopausia, } \\
\text { - Posición Socio/Económica**, } \\
\text { - Educación }\end{array}$ & $\begin{array}{l}\text { - HVI } \\
\text { - MicroAlbuminuria } \\
\text { - Creatinina > 1,3mg/dI } \\
\text { - IMT aumentado } \\
\text { - Retinopatía Hipertensiva } \\
\text { (grados III/IV) } \\
\text { - Aumento de rigidez vascular. }\end{array}$ & $\begin{array}{l}\text { - EC } \\
\text { - IM } \\
\text { - Stroke } \\
\text { - Arteriopatía periférica } \\
\text { - ICC } \\
\text { - ERC }\end{array}$ \\
\hline
\end{tabular}




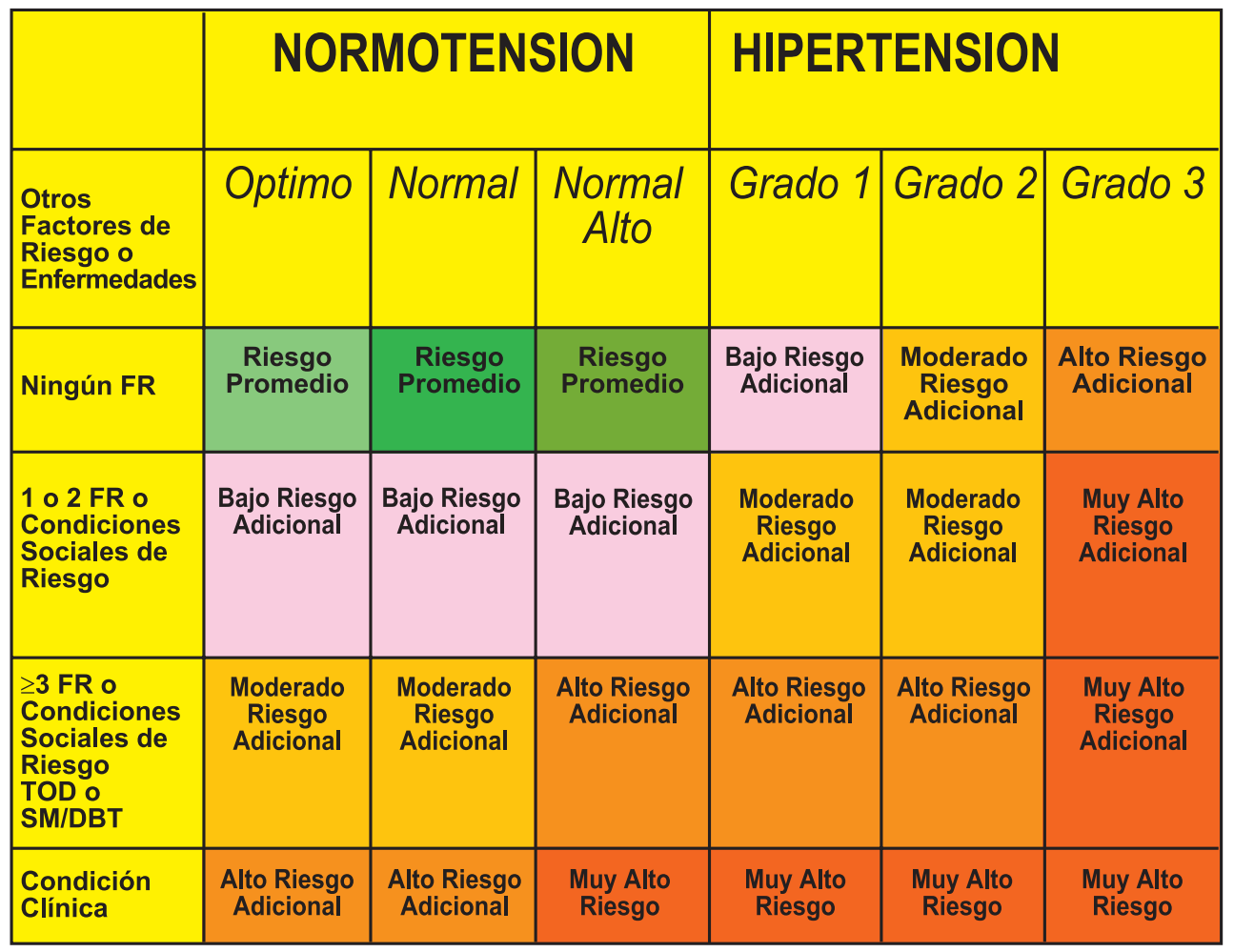

Figura 1: Riesgo relacionado con valores de presión arterial.

\section{Evaluación Diagnóstica del Sujeto Hipertenso}

El periodo de tiempo requerido para I evaluación inicial de un paciente hipertenso es de por lo menos 30 minutos. Los objetivos principales del diagnóstico se dirigen a:

1. Confirmar la existencia de cifras elevadas de presión arterial

2. Determinar el grado de hipertensión y la existencia de daño de órganos blanco

3. Evaluar la presencia de comorbilidades

4. Identificar tratamientos previamente recibidos o en uso actual

5. Cuantificar el riesgo global incluyendo sus componentes sociales

6. Diagnosticar o descartar posibles causas de hipertensión secundaria.

Historia Clínica y Examen Físico: No sólo se debe definir el grado de hipertensión, sino el momento en el cual de diagnosticó la hipertensión. Debe registrarse información acerca de la edad, sexo y raza. El examen físico debe incluir: medición de estatura, peso, cintura, cadera y cálculo de relación cintura a cadera e índice de masa corporal (IMC), la evaluación de los pulsos, frecuencia cardiaca, cifras de presión arterial, auscultación del corazón, búsqueda de soplos carotídeos, torácicos o periumbilicales, y un examen del fondo del ojo. Se debe buscar factores de riesgo asociados y posibles complicaciones, tales como edema periférico, angina de pecho, disnea, cefalea y latidos cardiacos ectópicos.

Las mediciones de la presión arterial deben ser realizadas de acuerdo con las recomendaciones de la American Heart Association, en dos posiciones diferentes (sentado y de pie), para poder descartar una posible hipotensión ortostática (disminución de más de $20 \mathrm{mmHg}$ en la sistólica y/o $10 \mathrm{mmHg}$ en la diastólica), especialmente frecuente en los pacientes mayores ${ }^{29}$. Cuando los valores de PAS y PAD 
R. Sánchez, M. Ayala, H. Baglivo, C. Velázquez, G. Burlando, O. kohlmann, et. al.

corresponden a grados diferentes, se debe usar el grado mayor para definir la hipertensión de ese paciente.

Las mediciones domiciliarias de la presión arterial, realizadas por personal entrenado con un esfigmomanómetro de mercurio, o preferiblemente un aparato automático o semiautomático validado, son una herramienta importante para el control y el seguimiento de los pacientes hipertensos. Los valores altos normales son similares para la PA domiciliaria y para el MAPA diurno, es decir $135 / 85 \mathrm{mmHg}^{30}$.

Pruebas de Laboratorio: Los principales objetivos son detectar otros factores de riesgo cardiovascular, evaluar daños a órganos blanco, e identificar causas secundarias de hipertensión. Siempre debe realizarse en la primera visita un hemograma, glicemia en ayunas, urea, creatinina sérica y en orina, electrolitos, ácido úrico, colesterol total, HDL y LDL, triglicéridos, pruebas de funcionalidad hepática, T3, T4 y TSH, además de un electrocardiograma, una orina completa, y una estimación de la velocidad de filtración glomerular (empleando creatinina sérica y la fórmula del estudio Modification of Diet in Renal Disease o MDRD [GFR $\left(\mathrm{mL} / \mathrm{min} / 1.73 \mathrm{~m}^{2}\right)=186 \times\left(\mathrm{S}_{\mathrm{cr}}\right)^{-1.154} \times(\text { edad })^{-0.203} \times(0.742$ en mujeres) $\times(1.212$ en afroamericanos] que puede calcularse en sitios específicos de la internet).

Exámenes recomendados: Se recomienda ecografía y Doppler vascular, cardiaco y renal para evaluar la masa ventricular izquierda y para identificar ateromatosis subclínica en los diferentes territorios vasculares, estenosis de arterias renales 0 alteraciones renales. La medición de la velocidad de la onda de pulso es útil para evaluar la rigidez de arterias grandes. Se recomienda con énfasis una microalbuminuria (en una muestra de orina de 24 horas o como relación albúmina/creatinina).

Medición Ambulatoria de la Presión Arterial (MAPA) ${ }^{31,32:}$ Este método, que no reemplaza a las mediciones

Figura 2: Algoritmo de Evaluación de la Presión Arterial

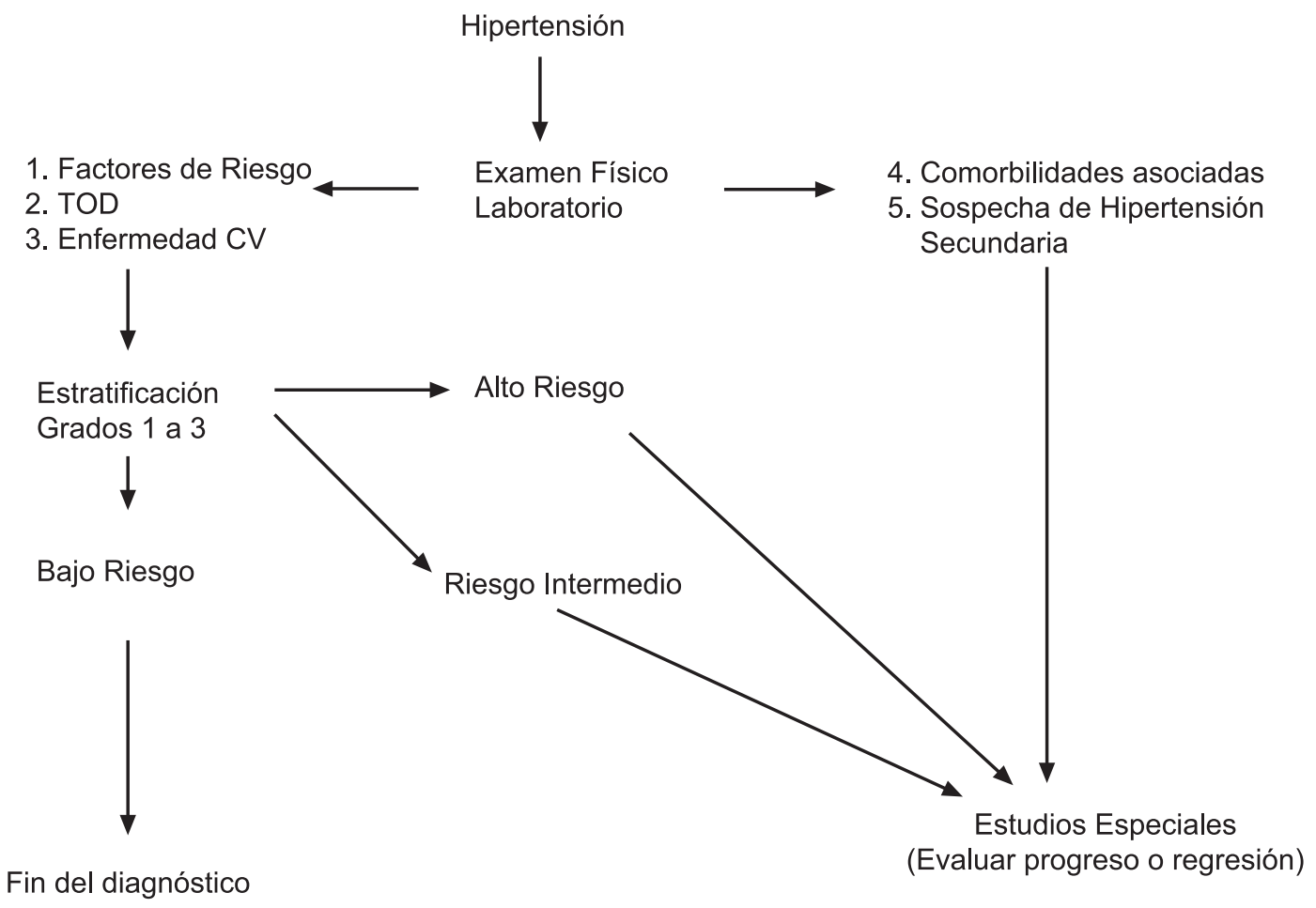


convencionales, da información detallada acerca de los valores promedios de día y de noche durante 24 horas. Los valores medios de 24 h están más cercanamente relacionados con el daño de órganos blanco y los resultados que los valores en consultorio.

EI MAPA está indicado cuando:

- se sospecha una hipertensión de la bata blanca

- se sospecha una hipertensión enmascarada u oculta

- la presión arterial es normal, pero acompaña un alto riesgo total

- es deseable la evaluación del perfil de PA de $24 \mathrm{~h}$ (dipping, non-dipping, etc.)

- se sospecha una hipertensión refractaria

- se buscan episodios de hipotensión o de hipertensión

- progresa, o no regresa, el daño de órganos blanco a pesar de un aparente buen control de la PA

Finalmente, el flujograma de la Figura 2 se puede seguir para evaluar a los sujetos hipertensos en los cuales se busca posibles causas de una hipertensión secundaria.

\section{Síndrome metabólico (SM), Diabetes Mellitus (DM1 O 2) e Hipertensión. Definición del sín- drome metabólico}

El sindrome metabólico es una entidad con características fácilmente detectables y con relevancia pronóstica, pero en gran medida sub-reconocida, que puede ser objeto de diagnóstico para reconocer a personas con riesgo cardiovascular elevado ${ }^{33-36}$. La Federación Internacional de Diabetes (International Diabetes Federation, IDF, https://www.idf.org/webdata/ docs/IDF_Meta_def_final.pdf) considera que la obesidad abdominal es uno de los principales factores de riesgo $\mathrm{CV}$. Sin embargo, como no hay datos acerca de los puntos de corte para la circunferencia abdominal y la grasa visceral en poblaciones Latinoamericanas, se recomienda usar datos de la población de Asia del Sur, hasta que haya datos más específicos disponibles. Algunos estudios pequeños apoyan esta propuesta ${ }^{37-39}$. Se está realizando estudios colaborativos y la nueva información puede arrojar información más fidedigna en este respecto. A pesar de que aún falta evidencia acerca de los beneficios de la intervención ${ }^{40}$, tiene sentido desde el punto de vista clínico y epidemiológico enfocarse en esta población para la prevención primaria de la diabetes mellitus.

Las definiciones de SM varían desde una definición estricta basada en la resistencia a la insulina (Organización Mundial de la Salud) a la que se basa solo en criterios clínicos (Programa Nacional de Educación acerca del Colesterol [National Cholesterol Education Program (NCEP) $]^{41}$. La definición del NCEP ha sido adoptada en pautas acerca de la hipertensión ${ }^{5}$, presumiblemente otorgando una mayor sensibilidad para identificar poblaciones de riesgo, pero al costo de una menor especificidad para la detección de una verdadera resistencia a la insulina ${ }^{5,6}$. Otra ventaja de la definición del NCEP es su sencillez clínica, pues se puede aplicar en casi cualquier lugar a pesar de los recursos limitados disponibles en Latinoamérica. Sin embargo, la definición de la IDF parece más apropiada para las poblaciones Latinoamericanas que las otras definiciones, con respecto a las diferencias étnicas ${ }^{37},{ }^{38}$. La prevalencia del SM varía con respecto a los criterios de clasificación, edad, sexo, raza, y estado socio-económico, pero es de aproximadamente 25 a $50 \%$ en Latinoamérica según criterios de la $\mathrm{IDF}^{37}$. Estimaciones recientes del valor pronóstico del SM mostraron relaciones de riesgo relativo para eventos cardiovasculares 0 mortalidad de 2.0 a 3.3 , incluyendo o no a la DM 42,43 .

\section{Definición de DM en Latinoamérica}

Hay cada vez más evidencia que apoya el concepto de que, tal como ocurre con la presión arterial,la glicemia en ayunas es una variable continua que aumenta el riesgo de enfermedad $\mathrm{CV}$, independientemente del hecho de haber alcanzado o no al punto de corte para el diagnóstico de diabetes ${ }^{44,45}$. Los criterios de diagnóstico de diabetes (glicemia en ayunas de al menos $126 \mathrm{mg} / \mathrm{dl}$ o glicemia de al menos $200 \mathrm{mg} / \mathrm{dl} 2$ horas después de la ingesta de una carga oral de glucosa) fueron elegidos porque identificaban individuos con alto riesgo de retinopatía. 
Recientemente se ha propuesto los términos disglicemia o pre-diabetes para definir una condición con glicemia en ayunas de 100 a 125.9 mg/dl o glicemia 2 horas después de la ingesta de una carga oral de $75 \mathrm{~g}$ de glucosa entre 140 y $199.9 \mathrm{mg} / \mathrm{dl}$. Se ha demostrado que el riesgo de desarrollar DM o ECV es mayor en sujetos con pre-diabetes ${ }^{46}$ sobre todo en poblaciones en países en vías de desarrollo ${ }^{14,47}$, que tienen propensión a desarrollar resistencia a la insulina asociada con adaptaciones epigenéticas la rápida transición económica y a los cambios del estilo de vida experimentados por estas poblaciones ${ }^{14,48}$.

La prevalencia poblacional de DM en Latinoamérica es de 5 a $9 \%$, siendo menor en zonas rurales y mayor en áreas con altitud por encima de $3000 \mathrm{~m}$, donde viven alrededor de 100.000 individuos. La prevalencia de la prediabetes es similar a la de DM. La prevalencia de hipertensión en la población diabética es 1,5 a 3 veces mayor que en los no diabéticos de la misma franja etaria ${ }^{6}$. La prevalencia de la hipertensión en diabetes tipo 1 es cercana al $30 \%$. Se desarrolla muchos años después del inicio de la diabetes, generalmente como consecuencia de la nefropatía diabética ${ }^{49}, 50$. Por otra parte, en la diabetes tipo 2 la hipertensión puede estar presente en el momento del diagnóstico o puede inclusive preceder a la hiperglicemia evidente ${ }^{51}$. En los diabéticos tipo 2 de diagnóstico reciente, la prevalencia de hipertensión es de aproximadamente $60 \%$. En la diabetes tipo 2 es difícil determinar si la hipertensión es secundaria a la diabetes, porque los pacientes generalmente son mayores y más frecuentemente obesos que los sujetos no diabéticos. Cabría esperar una mayor frecuencia de hipertensión, porque en las poblaciones occidentales la prevalencia de diabetes aumenta con la edad y con el grado de obesidad ${ }^{52}$. Sin embargo, después de ajustar por la edad y sobrepeso/obesidad, la prevalencia de hipertensión aún fue 1,5 veces mayor en los diabéticos que en los no diabéticos ${ }^{53}$. En ciertos grupos raciales ${ }^{54}$, la nefropatía diabética podría ser la causa primaria de hipertensión en los diabéticos de tipo 2, así como ocurre con los indios Pima y los afroamericanos. Los sujetos con diabetes e hipertensión corren riesgo de enfermedad macrovascular (coronaria, cerebrovascular y enfermedad vascular periférica) como microvascular (insuficiencia renal, retinopatía). Aunque la relación entre la neuropatía diabética y la hipertensión aun no está clara, cierta evidencia epidemiológica sugiere que la hipertensión puede facilitar el desarrollo de la neuropatía 6 .

\section{Complicaciones de la Diabetes}

Nefropatía diabética. La prevalencia de nefropatía es de 20 a $30 \%$ en pacientes con DM tipo 1, y 30 a $50 \%$ en pacientes con DM tipo $2^{55}$. Se describe tres estadíos:

Nefropatía incipiente, caracterizada por una velocidad de filtración glomerular (VFG) por encima de lo normal por uno 10 años, seguido por una excreción urinaria de albúmina aumentada (30 a $300 \mathrm{mg} /$ día) por unos 5 años. La presencia de una albuminuria elevada tanto en diabéticos tipo 1 como tipo 2 identifica a los pacientes que corren riesgo de enfermedad renal progresiva, y se considera un predictor independiente de enfermedad cardiovascular. Se reporta que el 20 a $40 \%$ de los individuos con microalbuminuria anormal progresan a una albuminuria franca, y el $20 \%$ a enfermedad renal terminal (End Stage Renal Disease o ESRD).

Nefropatía clínica, caracterizada por una excreción urinaria de albúmina de $>300 \mathrm{mg} /$ día e hipertensión en casi el $100 \%$ de los sujetos. En la DM tipo 1 el $80 \%$ de los pacientes pueden desarrollar una albuminuria $>300$ mg/día dentro de los 10 a 15 años, y muchos de ellos progresarán a ESRD. Sin intervención, el progreso a esta condición puede ser más rápido. De hecho, el $50 \%$ de los pacientes habrá llegado a ESRD dentro de los 10 años, y el $75 \%$ dentro de los 20 años $^{56}$. Por otra parte, las intervenciones terapéuticas en ambos tipos de DM pueden disminuir la velocidad de deterioro de la VFG.

La insuficiencia renal progresiva es macroalbuminuria (>300mg/día) y una VFG disminuida $(<30 \mathrm{ml} / \mathrm{min} x$ 
$1,73 \mathrm{~m}^{2}$ ). La macroalbuminuria identifica a los sujetos diabéticos con daño histológico sustancial y pronostica una disminución linear de la VFG. En los pacientes con DM tipo 2 de diagnóstico reciente se debe cuantificar la albuminuria anualmente. En los pacientes con DM tipo 1 se debe medir la albuminuria cada 5 a 7 años a partir del momento del diagnóstico, porque el aumento de la excreción urinaria de albúmina raramente ocurre más tempranamente en el curso de la enfermedad.

El manejo de la diabetes e hipertensión en pacientes nefrópatas justifica un control estricto de la glicemia y de la PA. La meta para la $\mathrm{HbA} 1 \mathrm{c}$ debe ser $<6,5$ a $7 \%$, porque esta ha demostrado disminuir el progreso de micro a macroalbuminuria ${ }^{5}$ El objetivo recomendado para el control de la PA es $<130 / 80 \mathrm{mmHg}, \mathrm{y}<125 / 70 \mathrm{mmHg}$ en los pacientes con proteinuria de $>1 \mathrm{~g} /$ día y/o disminución de la VFG.

Los ensayos randomizados de intervención han demostrado que el manejo de la hipertensión en los pacientes con nefropatía diabética debe incluir el bloqueo del sistema renina-angiotensina ${ }^{57-59}$ con agentes como los inhibidores de la enzima convertidora de angiotensina (IECAs, mayor evidencia en la DM tipo 1) o los bloqueadores de los receptores de angiotensina (BRAs, mayor evidencia en la DM tipo 2). Si no se logra la PA objetivo, debe agregarse otras drogas, como diuréticos (tiazidas), bloqueadores de calcio o beta bloqueantes. Frecuentemente se requiere combinar tres o más drogas para lograr la PA meta. Aunque hay datos a favor del uso combinado de IECAs y BRAs en pacientes con DM 2 y proteinuria, los resultados recientemente publicados acerca de esta combinación en el ensayo ONTARGET ${ }^{60}$ exigen cuidado, y se requiere una reevaluación cuidadosa de esta estrategia.

Los sujetos con nefropatía diabética requieren una restricción de la proteína en la dieta además del tratamiento farmacológico, porque una disminución de la ingesta de proteína $(0,6 \mathrm{~g} / \mathrm{kg}$ por día) puede disminuir la velocidad de la aparición de la enfermedad renal terminal ${ }^{61}$.

\section{Complicaciones cardiovasculares}

Los pacientes con hipertensión y diabetes corren mayor riesgo de enfermedades cardiovasculares (ECV) tales como enfermedad coronaria (EC), IC, AVE, y enfermedad vascular periférica. Las comorbilidades tales como la dislipidemia, el estado protrombótico y la disfunción autónoma, pueden contribuir a resultados no deseables, aumentando así la morbilidad y la mortalidad. La incidencia de la ECV en hombres y mujeres con DM 2 es hasta 3 a 4 veces mayor que en individuos no afectados. Es más, la DM está asociada con una tasa de mortalidad CV de más del $70 \%$, y la personas con DM 2 tienen 2 a 3 veces mayor probabilidad de morir de ECV que la personas sin antecedente de DM, aún después de controlar por otros factores de riesgo $\mathrm{CV}^{62-63}$. También corren alto riesgo de insuficiencia renal, amputación de miembros, deterioro cognitivo, muerte prematura, retinopatía que lleva a la ceguera, y disfunción eréctil.

Enfermedad coronaria. Varios factores son responsables del riesgo aumentado, entre los cuales niveles elevados de fibrinógeno (sobre todo durante un mal control de la glicemia), niveles elevados del inhibidor del activador de plasminógeno-1, y aumento de la agregación plaquetaria ${ }^{64}$. El tamizaje de EC debe incluir ergometría y estudios de perfusión miocárdica con SPECT, según necesidad.

El manejo de la EC es similar en los pacientes hipertensos con o sin DM. El dejar de fumar debe ser recomendado enfáticamente. Los propósitos del tratamiento incluyen el reestablecimiento del flujo coronario y perfusión miocárdica, estabilización de placas, prevención de isquemia recurrente, limitación del remodelamiento del VI, supresión de las arritmias, y prevención secundaria. El tratamiento debe incluir beta bloqueantes. El tratamiento antiplaquetario con 
R. Sánchez, M. Ayala, H. Baglivo, C. Velázquez, G. Burlando, O. kohlmann, et. al.

aspirina $^{65}$ es fundamental en el tratamiento de los pacientes diabéticos con EC, y también se recomienda durante y después de un infarto agudo de miocardio. Es importante lograr un control adecuado de la glicemia lo más tempranamente posible, porque los niveles de glicemia al ingreso son un predictor independiente de mortalidad precoz y tardía en los pacientes con infarto de miocardio ${ }^{66}$.

\section{Disfunción del ventrículo izquierdo e insuficiencia} cardíaca. La diabetes es un factor de riesgo mayor para la disfunción del ventrículo izquierdo (VI) y para IC. En el estudio Glasgow MONICA la incidencia de disfunción del VI fue mayor en pacientes diabéticos $(29 \%)$ en comparación con los no diabéticos $(7 \%)^{66}$. En el estudio de Framingham ${ }^{66}$, el riesgo relativo de IC clínica en los diabéticos fue 3,8 en hombres y 5,5 en mujeres, en comparación con los no diabéticos. Recientemente se informó una prevalencia de IC en sujetos diabéticos mayores de $39 \%{ }^{68}$. La tasa de IC se encontró ser 4,2/1000 pacientes/año para los pacientes diabéticos o con $\mathrm{HbA} 1 \mathrm{c}<7,0 \%$, y que aumentaba a 9,2/1000 pacientes/año para los que tenían $\mathrm{HbA} 1 \mathrm{c}>10 \%{ }^{69}$. Los pacientes diabéticos e hipertensos frecuentemente desarrollan lo que se denomina "insuficiencia cardiaca diastólica” - es decir IC con fracción de eyección sistólica conservada ${ }^{70}$. La alta prevalencia de IC y la significativa morbilidad y mortalidad asociadas justifican la identificación precoz de factores de riesgo y de signos clínicos que permitan el tratamiento apropiado. Aunque un electrocardiograma y radiografías pueden ser útiles, se recomienda ecocardiografía bidimensional y con Doppler pulsado cuando se sospecha IC, para visualizar los cambios en la estructura y la función del corazón subyacentes de la IC. También es importante el monitoreo con ECG de 24 horas para detectar arritmias, porque la IC es un conocido predictor de muerte súbita cardiaca.

Ensayos clínicos grandes han documentado los beneficios de drogas que bloquean los sistemas neurohormonales activados (simpático y renina-angiotensina) para atenuar el remodelamiento cardíaco, mejorar la función ventricular, y disminuir la morbilidad y mortalidad. El tratamiento debe incluir un diurético (furosemida), un IECA o BRA y un beta bloqueante, a menos que haya contraindicación. La espironolactona también puede ser considerada si no hay disfunción renal severa.

Accidente vascular encefálico. Las tasas de discapacidad relacionadas con los AVE son mayores en los diabéticos que en los no diabéticos ${ }^{71}$. El riesgo de AVE fatal versus no fatal está asociado con los niveles elevados de HbA1c muchos años antes del evento $^{72}$. Por ende, está justificado el control de PA y de glicemia, junto con otros tratamientos establecidos tales como la aspirina y las estatinas ${ }^{73}$ para la prevenirlos.

Prevención de enfermedad cardiovascular en pacientes diabéticos: el control de la glicemia y de la presión arterial es fundamental para la prevención de la enfermedad CV en pacientes con DM e hipertensión ${ }^{74,75}$. El Ensayo de Control de Diabetes y Complicaciones (Diabetes Control and Complications Trial, DCCT) en personas con diabetes tipo 1 encontró que un periodo de 6 años de control intenso de la glicemia (HbA1c 7,2\% vs 9,0\%) llevó a una disminución de $42 \%$ en complicaciones CV después de 11 o más años de seguimiento pasivo. Resulta interesante que durante el seguimiento pasivo no hubo diferencias en el control de la glicemia entre los dos grupos ${ }^{74}$. En la diabetes tipo 2 la evidencia es menos clara. El estudio UKPDS ${ }^{75}$ solo mostró beneficio para la prevención de enfermedad microvascular al disminuir $\mathrm{HbA} 1 \mathrm{c}$ a $7,0 \%$ vs $7,9 \%$, y en tres ensayos recientes (ACCORD ${ }^{76}$, ADVANCE $^{77}$ y DIGAMI $1-2^{78}$ el disminuir HbA1c a menos de $7 \%$ no mostró disminución significativa de a enfermedad macrovascular, aunque ADVANCE mostró una pequeña mejoría significativa en daños microvasculares. Sin embargo estos estudios indicaron que el riesgo CV de un sujeto diabético está directamente relacionado con la duración de la diabetes, y que la eficacia del tratamiento hipoglicemiante para disminuir 
el riesgo cardiovascular puede ser afectada por la duración de la diabetes (79). El tratamiento intensivo precoz debe ser recomendado en los sujetos diabéticos, sobre todo los que corren alto riesgo de desarrollar enfermedad CV, como son los sujetos diabéticos hipertensos.

\section{Riesgo de diabetes en hipertensión.}

La evidencia epidemiológica sugiere que la hipertensión es un factor de riesgo para el desarrollo de diabetes. Un estudio prospectivo encontró que persona con hipertensión tenían una incidencia de diabetes 2,4 veces mayor que personas no hipertensas ${ }^{80}$. Una explicación del riesgo mayor de diabetes en hipertensión es la activación del sistema reninaangiotensina. Tanto la vasoconstricción pancreática mediada por la angiotensina $\|^{81}$ y la hipokalemia mediada por la aldosterona ${ }^{82}$ inhiben la liberación de insulina inducida por glucosa de la célula beta. Además, la angiotensina II y la insulina comparten vías de transducción de señales. Así, la insulina activa a la proteinkinasa $C(P K C)$ a través de la fosforilación de tirosina del sustrato de receptor de insulina tipo 1 y tipo 2 (IRS-1, IRS-2) y estimula la vía de MAP-kinasa, mientras que la angiotensina II inhibe la señal de PKC, que altera la señal intracelular de insulina, produciendo una resistencia a la insulina ${ }^{83}$. El bloqueo del sistema renina-angiotensina disminuye a la hormona contrarreguladora norepinefrina ${ }^{84}$, mejora la sensibilidad periférica a la insulina ${ }^{85}$, y previene el desarrollo de diabetes en personas con hipertensión, cardiopatía o insuficiencia cardiaca, y disminuye los niveles de glicemia ${ }^{86-88}$. Por estos motivos las Pautas Europeas para el Manejo de la Hipertensión Arterial 2007 recomiendan que en pacientes hipertensos con sindrome metabólico y diabetes tipo 2 se deben usar IECA o BRA como primeras drogas antihipertensivas. En las poblaciones latinoamericanas se debe hacer recomendaciones similares para este tipo de pacientes, sobre todo porque son más propensos a desarrollar resistencia a la insulina a menores niveles de obesidad abdominal ${ }^{89,90}$, una condición con características epidémicas en Latinoamérica91, asociada con cambios en la función vascular independientemente de otros factores de riesgo cardiovascular ${ }^{92}$.

\section{TRATAMIENTO DE LA HIPERTENSIÓN: (Figura 3)}

\section{Principios Generales}

Regiones de ingresos medianos y bajos, como la mayoría de los países latinoamericanos, tienen una carga de enfermedad cinco veces mayor que los países de altos ingresos, con acceso a menos del $10 \%$ de los recursos globales de tratamiento. Por ende, se debe dar prioridad a los que corren mayor riesgo de eventos fatales, porque la mayoría de los pacientes hipertensos no reciben ningún tratamiento. Debe prestarse atención especial a los individuos con condiciones de riesgo social, tales como las personas sin hogar, los pobres, deficientes en educación o desempleados.

En los pacientes hipertensos, el propósito primario del tratamiento es lograr la mayor reducción en el riesgo CV total a largo plazo, manteniendo una buena calidad de vida. Esto requiere tratamiento de los valores elevados de PA, como también de todos los factores de riesgo asociados reversibles para disminuir el riesgo CV asociado. Así, cualquier reducción en la PA, aunque no sea óptima, ayuda a disminuir el riesgo total. Sin embargo, la PA se debe disminuir por lo menos a 140/90 mmHg (sistólica/diastólica), y a cifras aún menores según tolerancia, en todos los pacientes hipertensos. La PA meta debe ser al menos $<130 / 80 \mathrm{mmHg}$ en pacientes con diabetes y en pacientes con riesgo alto o muy alto, tales como lo que tienen condiciones clínicas asociadas (ACV, infarto de miocardio, disfunción renal, proteinuria) $)^{5,} 93$.

La PA sistólica es mejor predictor de riesgo en pacientes ancianos. También en estos pacientes la meta del tratamiento debe ser lograr $<140 \mathrm{mmHg}$. En los hipertensos muy ancianos se encontró una reducción importante del riesgo $\mathrm{CV}$ en el estudio HYVET con una PA meta de 150/80 $\mathrm{mmHg}^{94}$. A pesar 
R. Sánchez, M. Ayala, H. Baglivo, C. Velázquez, G. Burlando, O. kohlmann, et. al.

del uso de tratamiento combinado, puede ser difícil alcanzar una PA sistólica $<140 \mathrm{mmHg}$, y aún más si la meta es bajar a $<130 \mathrm{mmHg}$. Se puede esperar más dificultades en los ancianos, en los pacientes con diabetes, y en general en pacientes con daño CV. ,104
Para lograr más fácilmente la PA deseada, el tratamiento antihipertensivo se debe iniciar antes de que se produzca daño CV significativo. El monitoreo ambulatorio de la PA durante $24 \mathrm{~h}$ es una herramienta útil que se debe recomendar, si está disponible, para reforzar o corregir el tratamiento ${ }^{95-98}$.

\begin{tabular}{|c|c|c|c|c|c|c|}
\hline \multirow[b]{2}{*}{$\begin{array}{l}\text { PA } \\
\text { Factores de } \\
\text { Riesgo CV }\end{array}$} & \multicolumn{3}{|c|}{ Normotensión } & \multicolumn{3}{|c|}{ Hipertensión } \\
\hline & $\begin{array}{c}\text { Óptima } \\
<12 / 80 \mathrm{mmHg}\end{array}$ & $\begin{array}{c}\text { Normal } \\
120-134 / 80-84 \mathrm{mmHg}\end{array}$ & $\begin{array}{c}\text { Normal alta } \\
135-139 / 85-89 \mathrm{mmHg}\end{array}$ & $\begin{array}{c}\text { Grado } 1 \\
140-149 / 90-99 \text { mmHg }\end{array}$ & $\begin{array}{c}\text { Grado } 2 \\
160-179 / 100-109\end{array}$ & $\begin{array}{c}\text { Grado } 3 \\
\geq 180 / 110 \mathrm{mmHg}\end{array}$ \\
\hline Ninguno & $\begin{array}{c}\text { Ninguna } \\
\text { Intervención }\end{array}$ & $\begin{array}{c}\text { Ninguna } \\
\text { Intervención }\end{array}$ & $\begin{array}{l}\text { Cambios de } \\
\text { Estilo de vida }\end{array}$ & $\begin{array}{l}\text { Cambios de } \\
\text { Estilo de vida y } \\
\text { trataniento } \\
\text { farmacológico }\end{array}$ & $\begin{array}{l}\text { Cambios de } \\
\text { Estilo de vida y } \\
\text { trataniento } \\
\text { farmacológico }\end{array}$ & $\begin{array}{l}\text { Cambios de } \\
\text { Estilo de vida y } \\
\text { trataniento } \\
\text { farmacológico }\end{array}$ \\
\hline $\begin{array}{l}\text { 1-2 y/o } \\
\text { Condiciones } \\
\text { de Riesgo } \\
\text { Social }\end{array}$ & $\begin{array}{l}\text { Cambios de } \\
\text { Estilo de vida }\end{array}$ & $\begin{array}{l}\text { Cambios de } \\
\text { Estilo de vida }\end{array}$ & $\begin{array}{l}\text { Cambios de } \\
\text { Estilo de vida y } \\
\text { trataniento } \\
\text { farmacológico }\end{array}$ & $\begin{array}{l}\text { Cambios de } \\
\text { Estilo de vida y } \\
\text { trataniento } \\
\text { farmacológico }\end{array}$ & $\begin{array}{l}\text { Inicio inmediato } \\
\text { de tratamiento } \\
\text { farmacológico }\end{array}$ & $\begin{array}{l}\text { Inicio inmediato } \\
\text { de tratamiento } \\
\text { farmacológico }\end{array}$ \\
\hline \begin{tabular}{|l|}
$\geq 3$ y \\
Condiciones \\
de riesgo \\
Social \\
\end{tabular} & $\begin{array}{l}\text { Cambios de } \\
\text { Estilo de vida }\end{array}$ & $\begin{array}{l}\text { Cambios de } \\
\text { Estilo de vida }\end{array}$ & $\begin{array}{c}\text { Cambios de } \\
\text { Estilo de vida y } \\
\text { trataniento } \\
\text { farmacológico }\end{array}$ & $\begin{array}{l}\text { Cambios de } \\
\text { Estilo de vida y } \\
\text { trataniento } \\
\text { farmacológico }\end{array}$ & $\begin{array}{l}\text { Inicio inmediato } \\
\text { de tratamiento } \\
\text { farmacológico }\end{array}$ & $\begin{array}{l}\text { Inicio inmediato } \\
\text { de tratamiento } \\
\text { farmacológico }\end{array}$ \\
\hline $\begin{array}{l}\text { Enfermedad } \\
\text { Clínica } \\
\text { Asociada }\end{array}$ & $\begin{array}{c}\text { Cambios de } \\
\text { Estilo de vida y } \\
\text { trataniento } \\
\text { farmacológico }\end{array}$ & $\begin{array}{l}\text { Cambios de } \\
\text { Estilo de vida y } \\
\text { trataniento } \\
\text { farmacológico }\end{array}$ & $\begin{array}{c}\text { Cambios de } \\
\text { Estilo de vida y } \\
\text { trataniento } \\
\text { farmacológico }\end{array}$ & $\begin{array}{l}\text { Inicio inmediato } \\
\text { de tratamiento } \\
\text { farmacológico }\end{array}$ & $\begin{array}{l}\text { Inicio inmediato } \\
\text { de tratamiento } \\
\text { farmacológico }\end{array}$ & $\begin{array}{l}\text { Inicio inmediato } \\
\text { de tratamiento } \\
\text { farmacológico }\end{array}$ \\
\hline
\end{tabular}

Figura 3: Intervensiones Terapéuticas relacionadas con Valores de Pa y Riesgo

\section{Cambios del Estilo de Vida}

Debe instituirse medidas de estilo de vida cada vez que sea apropiado en todo los pacientes hipertensos, incluyendo a los que requieren tratamiento farmacológico. El propósito es bajar la PA, controlar otros factores de riesgo, y disminuir el número o las dosis de drogas antihipertensivas. También son recomendables medidas de estilo de vida en sujetos con presión arterial normal y normal-alta (véase Fig. 3) para disminuir el riesgo de desarrollar hipertensión. Las recomendaciones de estilo de vida no deben ser dadas como una formalidad, sino que deben ser instituidas con suficiente apoyo de expertos y de comportamiento, con refuerzos periódicos. Las medidas de estilo de vida que son ampliamente conocidas que bajan la $\mathrm{PA}$ y/o el riesgo $\mathrm{CV}$ y que se debe considerar son:

- dejar de fumar

- bajar de peso (y estabilizar el peso)

- disminuir el consumo excesivo de alcohol

- ejercicio físico

- disminución del consumo de sal $(<6 \mathrm{~g} \mathrm{NaCl})$

- aumentar el consumo de $\mathrm{K}+(>6 \mathrm{~g})$

- aumentar el consumo de frutas y verduras y disminuir el consumo de grasas saturadas y totales.

El IMC y la circunferencia abdominal son marcadores clínicos fidedignos en la prevención CV. El IMC óptimo para a población hipertensiva es entre 18,5 y 
$25 \mathrm{Kg} / \mathrm{m} 2$. Del mismo modo, una circunferencia abdominal adecuada es $<90 \mathrm{~cm}$ en los hombres y $<80 \mathrm{~cm}$ en las mujeres (37-39), pero no hay tablas de valores normales basados en estudios epidemiológicos de suficiente poder disponible en Latinoamérica.

El ejercicio aeróbico es un complemento importante de la dieta para la reducción del peso y de la PA. Debe ser implementado en todos los hipertensos, y especialmente en los que tiene otros factores de riesgo, por al menos 30 minutos diarios.

Porque el cumplimiento a largo plazo con las medidas de estilo de vida es bajo, y porque la respuesta de la PA a estas es muy variable, debe hacerse un seguimiento cercano de los pacientes tratados con medidas no farmacológicas.

\section{Inicio del tratamiento para bajar la PA}

El inicio del tratamiento para bajar la PA debe decidirse según dos criterios: el nivel de la PAS y de la PAD y el nivel de riesgo CV total. El tratamiento farmacológico debe ser iniciado inmediatamente en la hipertensión grado 3 así como en la grado 1 y 2 cuando el riesgo CV total es alto o muy alto. En los hipertensos de grado $1 \circ 2$ con riesgo CV total moderado se puede postergar el tratamiento farmacológico por unas semanas, y en los hipertensos grado 1 sin otro factor de riesgo se puede postergar por varios meses. Sin embargo, es importante prestar atención muy especial a los individuos que corren riesgo a causa de su ambiente social (sin hogar, pobres, no educados o desempleados) en los cuales se debe considerar el valor de iniciar el tratamiento más rápidamente y en quienes un seguimiento cercano de la salud es obligatorio. Cuando la presión arterial inicial está en el rango alto-normal, la decisión de la intervención farmacológica depende en gran medida de la condición clínica individual. En el caso de diabetes, antecedentes de enfermedad cerebrovascular, coronaria o arterial periférica, la recomendación de iniciar fármacos para bajar la presión arterial se justifica por los resultados de ensayos controlados. A los sujetos con presión arterial normal pero con riesgo cardiovascular muy alto a causa de una patología clínica asociada se debe recomendar medidas intensas de estilo de vida. En estos sujetos la presión arterial debe ser monitorizada cuidadosamente, y se debe considerar tratamiento farmacológico en presencia de presión arterial que va en aumento o empeoramiento de la condición clínica.

\section{Selección de drogas antihipertensivas}

Los principales beneficios del tratamiento antihipertensivo se deben a la mejoría de la PA per se. Cinco clases mayores de agentes antihipertensivos son aptos para el inicio y el mantenimiento del tratamiento antihipertensivo, solos o en combinación ${ }^{5}$ : diuréticos tiazidas, bloqueantes del calcio, inhibidores de la ECA (IECA), bloqueantes del receptor de angiotensina (BRA) y beta bloqueantes. Los beta bloqueantes, sobre todo en combinación con un diurético tiazida, no se debe usar en pacientes con el sindrome metabólico o con alto riesgo de incidencia de diabetes. En estos pacientes, el carvedilol, nebivolol o indapamida de liberación lenta pueden ser aptos ${ }^{99-101}$. Los inhibidores de la renina, tales como el aliskiren, aunque no disponibles en todos los países, han demostrado ser efectivos como agentes antihipertensivos ${ }^{102}$; sin embargo, aún se aguardan los resultados de algunos ensayos, y todavía se desconoce la relación costo/beneficio de estos agentes. En muchos pacientes se necesita más de una droga, así que combinaciones fijas pueden ser útiles para mejorar el cumplimiento y aumentar el éxito en el control de la presión arterial ${ }^{103}$.

La elección de la droga específica, o la combinación de drogas, y el evitar otras debe tener en cuenta lo siguiente:

1. Laexperiencia previadel paciente individual, favorable o no favorable, con cierta clase de antihipertensivos.

2. ElefectodelasdrogassobrelosfactoresderiesgoCVen relaciónconel perfil de riego $\mathrm{CV}$ del paciente individual.

3. La presencia de daño subclínico de órganos, enfermedad CV clínica, enfermedad renal o diabetes, 
R. Sánchez, M. Ayala, H. Baglivo, C. Velázquez, G. Burlando, O. kohlmann, et. al.

que puede ser tratada más favorablemente por algunas drogas que por otras.

4. La presencia de otros trastornos que pueden limitar el uso de ciertas clases de drogas antihipertensivas.

5. Las posibilidades de interacciones con drogas usadas por otras co-morbilidades.

6. El costo de la drogas, ya sea para el individuo o el proveedor de salud. Sin embargo, las consideraciones de costos nunca deben predominar con respecto a la eficacia, tolerabilidad, y la protección del paciente individual.

Debe prestarse atención continuamente a los efectos colaterales de las drogas, porque estos son la causa más importante del no-cumplimiento. Las drogas no son equivalentes en términos de los efectos adversos, sobre todo en pacientes individuales. Las drogas que ejercen su efecto antihipertensivo durante 24 horas con una sola administración diaria deben ser preferidas porque una posología sencilla favorece el cumplimiento ${ }^{104}$.

En los pacientes hipertensos con riesgo CV moderado $\mathrm{o}$ alto y condiciones específicas acompañantes, se recomienda las siguientes intervenciones farmacológicas:

1. IECAs o BRAs en pacientes con sindrome metabólico o diabetes tipo 2 porque los parámetros metabólicos no son afectados o pueden incluso mejorar.

2. IECAs o BRAs en pacientes con disfunción renal y microalbuminuria o proteinuria porque estos agentes enlentecen el progreso a la insuficiencia renal crónica y diálisis.

3. IECAs o BRAs en pacientes con disfunción ventricular izquierda sistólica y diastólica, aunque sea asintomática.

4. IECAs o BRAs y bloqueadores de los canales de calcio en pacientes con hipertrofia ventricular izquierda porque estos agentes facilitan la regresión ventricular izquierda.

5. Beta bloqueantes en pacientes con EC.

6. Bloqueantes de canales de calcio (dihidropiridinas) en hipertensos adultos mayores y en hipertensos afroamericanos.
7. Bloqueantes alfa adrenérgicos en pacientes con hipertrofia prostática.

8. Tiazidasyclortalidonaen hipertensosafroamericanos, hipertensos adultos mayores o personas de bajos ingresos que no tienen acceso a otras drogas más costosas.

9. En hipertensos con insuficiencia cardíaca, diuréticos, IECAs, carvedilol o nebivolol, y espironolactona

10. En pacientes post infarto de miocardio IECAs y beta bloqueantes

11. La recidiva de los AVE se previene mejor con diuréticos (indapamida de liberación lenta) e IECA.

12. Los pacientes con enfermedad vascular periférica deben dejar de fumar y realizar ejercicios aeróbicos. Los bloqueantes de los canales de calcio son adecuados para bajar la presión arterial sin exacerbar los síntomas.

13. IECAs o BRAs en pacientes con fibrilación auricular recidivante. Beta bloqueantes o verapamilo en fibrilación auricular sostenida.

14. Estatinas y drogas antiplaquetarias en hipertensos de muy alto riesgo (prevención secundaria).

\section{POBLACIONES ESPECIALES}

\section{HIPERTENSIÓN EN NIÑOS:}

\section{Definición de hipertensión en niños}

La hipertensión en pediatría se define siguiendo tablas de percentilos relacionados con el sexo, edad y el peso, del Informe del Grupo de Trabajo sobre la Presión Arterial Alta en Niños y Adolescentes ${ }^{105}$. Se define como presión arterial normal cuando la presión arterial, según sexo, edad y peso, está por debajo del percentilo 90, presión alta-normal cuando está por encima del percentilo 90, pero por debajo del percentilo 95 , en 3 ocasiones o más.

Los adolescentes con presión de $\geq 120 / 80$ deben ser considerados pre-hipertensos. La hipertensión de bata blanca se define cuando los valores son iguales o mayores al percentilo 95, y en rango normal fuera del consultorio. Para la confirmación diagnóstica se requiere MAPA o mediciones domiciliarias de la PA. 
Medición de la presión arterial: La relación demostrada entre los valores de la presión arterial en niños y la hipertensión esencial en la vida adulta apoya la recomendación de que la presión arterial debe ser controlada de forma rutinaria en el examen físico pediátrico. El viejo concepto que la hipertensión en niños y adolescentes es predominantemente secundaria es incorrecto. Así, se recomienda que la PA se debe medir desde los primeros días de vida y al menos una vez al año incluso en niños, sobre todo los obesos, porque la hipertensión está muy relacionada con la obesidad en niños ${ }^{106-111}$.

La medición de la presión arterial es obligatoria en enfermedades o condiciones acompañadas por un mayor riesgo de hipertensión arterial, tales como las enfermedades renales, diabetes, resistencia a la insulina, corticoterapia a largo plazo, antiinflamatorios no esteroidales, anticonceptivos orales, ciclosporina, neurofibromatosis, neonatos con vasos umbilicales patológicos, sindrome de Turner, coartación de aorta corregida, sindrome hemolítico-urémico, insuficiencia cardíaca sin explicación, miocardiopatía dilatada y convulsiones de etiología desconocida.

Las mediciones de la presión arterial deben seguir estas recomendaciones:

a) El niño debe estar sentado en una silla que le permite tener apoyo para el brazo. Si esto no es posible, el niño debe estar sentado en el regazo de la madre.

b) Ninguna medición de la presión arterial se debe hacer si el niño está llorando o moviendo el brazo en el cual se medirá la presión.

c) Para elegir el manguito adecuado, se debe medir la circunferencia a la mitad de la distancia entre el acromión y el olécranon (Tabla 5).

Tabla 5: Largo del manguito recomendado para niños y adolescentes ${ }^{9}$

\begin{tabular}{lccc}
\hline & \multicolumn{2}{c}{ Vejiga Inflada } & Manguito Flexible \\
Largo $(\mathrm{cm})$
\end{tabular}

*calculo para cubrir al menos el $80 \%$ de la circunferencia del brazo

La vejiga, y no el manguito, debe cubrir el $80 \%$ de la circunferencia del brazo y $2 / 3$ de la distancia olécranon-acromión. Si el manguito correcto no está disponible, puede usarse el siguiente tamaño. Si solo se dispone de un manguito para adultos, se puede usar el muslo del niño, con el niño en decúbito, auscultando en la fosa poplítea. A partir de los $6 \circ 7$ años puede usarse un manguito pequeño para adultos.

Evaluación diagnóstica: Los niños con hipertensión arterial marcada y persistente deben ser evaluados para buscar una hipertensión secundaria. Debe averiguarse acerca de enfermedades neonatales y antecedentes de infecciones urinarias, hábitos alimentarios, bebidas energizantes y drogas ilegales. El examen físico debe incluir la frecuencia cardíaca, pulsos periféricos, soplos vasculares y cardíacos, y presión arterial en ambos brazos y piernas. El MAPA o la PA domiciliaria pueden ser útiles para confirmar el diagnóstico y descartar hipertensión de bata blanca, frecuente en adolescentes ${ }^{112,113}$, evitando así estudios innecesarios. La clasificación de MAPA de $24 \mathrm{~h}$ 
R. Sánchez, M. Ayala, H. Baglivo, C. Velázquez, G. Burlando, O. kohlmann, et. al.

relacionado con el género y la edad puede realizarse usando tablas que aparecen en otras publicaciones ${ }^{114}$.

Cuando se sospecha consistentemente una hipertensión secundaria debe realizarse ecocardiografía y Doppler renal, hemograma; en suero, creatinina, ácido úrico, evaluación ácido-base, actividad plasmática de renina, aldosterona sérica, glicemia en ayunas, y perfil lipídico; y en orina, creatinina, electrolitos y orina completa, con microalbuminuria.

Si se confirma la sospecha, se puede considerar hacer pruebas especiales según el tipo de hipertensión secundaria, entre las cuales cintigrafía renal antes y después de IECA, angio-resonancia magnética $y / 0$ arteriografía aorto-renal (enfermedad renovascular), metanefrina y catecolaminas urinarias (feocromocitoma), relación de aldosterona:renina y niveles de cortisol en plasma (hiperaldosteronismo primario o enfermedad de Cushing).

Prevención: Los pediatras deben medir la presión arterial como parte del examen físico en todos los niños. Los médicos encargados de adultos hipertensos o adultos cardiópatas también deben medir la presión arterial en los hijos de sus pacientes, porque la hipertensión muestra agregación familiar ${ }^{115}$. Los cambios de estilo de vida deben ser recomendados para toda la familia, contribuyendo así en gran medida a la prevención primaria de la hipertensión y de la morbi-mortalidad cardiovascular. Debe promocionarse cambios saludables en la dieta y aumento de la actividad física en las escuelas y en los colegios, para combatir activamente la epidemia de exceso de peso y obesidad inducida por la ingesta de comida chatarra y por el sedentarismo debido al tiempo pasado frente a la computadora y de la televisión. La obesidad es uno de los principales componentes del síndrome metabólico que también incluye otros factores de riesgo cardiovascular, tales como la hipertensión arterial, la resistencia a la insulina y la dislipidemia ${ }^{108,}{ }^{116}$. La ingesta elevada de sal a través de las comidas proce- sadas y el consumo de azúcar en bebidas endulzadas también contribuyen al aumento del peso ${ }^{117}$. Una disminución de la ingesta de sal es una medida simple para prevenir el aumento de peso. Un mayor consumo de frutas y verduras, comidas con alto contenido de potasio, también puede prevenir un aumento de la presión arterial. Debe recomendarse no exponerse al humo de tabaco ni de forma activa ni pasiva, pues hay una relación linear entre el tabaquismo de los padres y de los niños. La infancia es una ventana específica en la cual se debe iniciar la prevención de factores de riesgo para la hipertensión y cardiovasculares.

Tratamiento: El tratamiento no farmacológico es la medida principal para bajar la presión arterial en niños. Es parecido a lo que se recomienda más arriba para la prevención de la hipertensión.

El tratamiento farmacológico no debe iniciarse antes de 4 a 6 meses de respuesta no satisfactoria a los cambios del estilo de vida, cuando hay daño de órganos blanco y en sujetos con hipertensión secundaria.

Los estudios clínicos con drogas antihipertensivas en niños no son numerosos ni grandes, pero han dado algo de información acerca de las dosis y de la seguridad de las drogas. El tratamiento debe iniciarse con una droga única, siendo adecuadas todas las clases (IECAs, BRAs, beta bloqueantes, antagonistas del calcio y diuréticos). Inicialmente debe usarse dosis pequeñas, y luego titularse hasta lograr control de la presión arterial. Si esto fracasa, puede agregarse una segunda droga para evitar dosis muy elevadas de una droga cualquiera.

\section{Precauciones en el tratamiento de la hipertensión} en niños: Evitar el uso de IECAs y de BRAs en niñas adolescentes con riesgo de embarazo (contraindicación específica). Los beta bloqueantes pueden causar algo de reducción del rendimiento físico y los diuréticos aumentan el riesgo de disturbios electrolíticos. 


\section{HIPERTENSIÓN EN EL EMBARAZO}

En el embarazo la hipertensión tiene una prevalencia de aproximadamente 5 a $10 \%$, y es más prevalente en los embarazos de alto riesgo, como los que tienen antecedentes de pre-eclampsia o hipertensión crónica severa, o en primíparas. En Latinoamérica se ha documentado una prevalencia mayor de hipertensión en el embarazo que en países de altos ingresos ${ }^{118}$. Por ende, debe prestarse atención especial a este tema en las presentes pautas. De hecho, la mayoría de las complicaciones de la hipertensión en mujeres embarazadas son prevenibles, y la mejor prevención se basa en la detección precoz de la hipertensión a través de un monitoreo cuidadoso de la presión arterial.

Definición y clasificación: La hipertensión en el embarazo se define como valores de presión arterial $\geq 140 / 90 \mathrm{mmHg}$ en dos o más mediciones con un intervalo de 4 horas $^{119}$. La proteinuria en el embarazo se define como una excreción de proteína de $\geq 300 \mathrm{mg} /$ 24h. Entre los diferentes sindromes hipertensivos en el embarazo se llama la atención especial a la preeclampsia a causa de su prevalencia en Latinoamérica.

La pre-eclampsia frecuentemente está asociada con complicaciones fetales. Se inicia con una placentación anómala antes de la semana $20^{120}$, y generalmente se presenta clínicamente después de la semana 28, con un aumento en la presión arterial, proteinuria e hiperuricemia. También puede haber edema, disminución de la función renal, hemólisis y agregación plaquetaria, atribuida a una disfunción endotelial secundaria a isquemia placentaria. La disfunción endotelial puede liberar diferentes toxinas a la sangre materna, tales como citoquinas, especies reactivas de oxígeno (reactive oxygen species, ROS), dimetilarginina asimétrica (ADMA, asymmetric dimethylarginine), anticuerpos contra los receptores de angiotensina II, etc. ${ }^{121}$. Factores que favorecen la placentación anormal son: primer embarazo antes de los 18 años o después de los 40 años de edad, antecedente de pre-eclampsia (especialmente si ocurrió antes de la semana $32^{122}$, antecedente de aborto espontáneo o de retraso severo del crecimiento intrauterino, embarazo que resulte de fertilización asistida, embarazos múltiples, antecedente familiar cercano (madre, hermana) de pre-eclampsia, isoinmunización $\mathrm{Rh}$, infecciones subclínicas, hipertensión arterial crónica, insuficiencia renal, obesidad, enfermedades autoinmunes. Se ha descrito diferencias entre países desarrollados en las causas de pre-eclampsia y en las estrategias par prevenirla ${ }^{123}$ La inflamación secundaria a infecciones vaginales y urinarias subclínicas ${ }^{124,125}$, enfermedad periodontal ${ }^{126}$, resistencia a la insulina ${ }^{127}$ son considerados posibles factores de riesgo para el desarrollo de la pre-eclampsia. Por ende, es importante que las mujeres embarazadas con estos factores de riesgo sean estudiadas cuidadosamente para detectar precozmente hipertensión y proteinuria, prevenir complicaciones severas que requieren hospitalización, y, si fuere necesario, realizar una cesárea para preservar a la madre y al niño.

\section{Prevención}

Dosis bajas de aspirina: Su eficacia es menor que la esperada inicialmente ${ }^{128}$ y su uso sigue siendo controvertido. Sin embargo, en pacientes de alto de pre eclampsia, la administración precoz de aspirina (100mg/día desde la 8 semana hasta 2 semanas antes del probable parto) puede retardar el inicio de la pre-eclampsia.

Suplementación con calcio: Se ha demostrado una relación inversa entre la ingesta de calcio y la preeclampsia' ${ }^{129}$. Aunque varios ensayos de suplementación de calcio no han logrado mostrar beneficio consistentemente, algunas poblaciones de bajos y medianos ingresos en América Latina consumen poco calcio en la dieta, y en estos grupos se ha logrado efectos beneficiosos sobre la preeclampsia y los partos prematuros con la administración diaria de $1 \mathrm{~g}$ de calcio ${ }^{130-132}$.

Detección de infecciones subclínicas: A causa de la fuerte correlación entre las infecciones urinarias y periodontales y la preeclampsia ${ }^{133}$, es de importancia capital buscar y tratar la bacteriuria, y las infecciones urinarias, vaginales y periodontales en las mujeres embarazadas. 


\section{Estudios de diagnóstico}

Las pruebas bioquímicas durante el embarazo deben incluir hemograma, glicemia, electrolitos séricos, creatinina y ácido úrico, análisis de orina y proteinuria de 24 hs. Estas determinaciones deben repetirse a las semanas $20,28,32$ y 36 , y más frecuentemente en caso de hipertensión o de complicaciones. En embarazadas con alto riesgo de preeclampsia es recomendable realizar Doppler de arterias uterinas a las semanas 10-12 y 20-28.

Las anomalías de la coagulación son dos a tres veces más frecuentes en mujeres que tienen antecedentes de preeclampsia antes de la semana 32 , o de abortos recurrentes, que en la población general ${ }^{134-135}$. Una búsqueda de anticuerpos anticardiolipina, homocisteína, déficit de antitrombina III, inhibidor lúpico, resistencia a la proteína $C$ reactiva, déficit de proteína $\mathrm{S}$, ayuda a identificar mujeres que en embarazos ulteriores pueden requerir aspirina o heparina para prevenir las complicaciones. Del mismo modo, se debe hacer una curva de glicemia en toda mujer embarazada con glicemia en ayunas elevada.

\section{Tratamiento}

El tratamiento farmacológico se debe iniciar cuando la presión arterial es $\geq 150 / 100 \mathrm{mmHg}$. El tratamiento se puede iniciar por vía oral, y se puede volver a controlar a la paciente a las 48 o $72 \mathrm{hs}$.

Basado en 40 años de experiencia y, en 7,5 años de seguimiento de hijos de madres tratadas, la alfametil dopa (500 a 2000 mg/día) es la droga de primera elección. Entre las drogas de segunda línea se encuentran el labetalol (100 a $400 \mathrm{mg} / \mathrm{día}$ ), la nifedipina de larga acción (30 a 60 mg/día) y la hidralazina (50 a $200 \mathrm{mg} / \mathrm{día})^{136}$.

Tienen contraindicación absoluta los inhibidores de la renina, los IECA y los BRA ${ }^{137-138}$. Tienen contraindicación relativa: los beta bloqueantes (sobre todo el atenolol), a causa de perfusión placentaria disminuida y efectos indeseables en los neonatos (peso disminuido, bradicardia e hipoglicemia) ${ }^{139}$. Los diuréticos también están relativamente contraindicados, a menos que haya insuficiencia cardiaca, porque la preeclampsia se caracteriza por un volumen plasmático disminuido, que puede ser disminuido aún más por el tratamiento con diuréticos.

Se requiere una internación y tratamiento de urgencia (frecuentemente tratamiento endovenoso) si la presión arterial diastólica es $\geq 110 \mathrm{~mm} \mathrm{Hg} \mathrm{o}$ permanece $>100 \mathrm{~mm} \mathrm{Hg}$ a pesar de tratamiento, si la proteinuria es $>1 \mathrm{~g} / 24 \mathrm{~h}$, si hay sindrome de HELLP o eclampsia. Aún en las situaciones de urgencia, la presión arterial se debe disminuir lentamente durante las primeras $24 \mathrm{hs}$.

Las drogas recomendadas son: Labetalol: dosis inicial $20 \mathrm{mg}$ iv, con dosis subsecuentes cada $10 \mathrm{~min}$, si necesario, hasta una dosis máxima de $220 \mathrm{mg}$; Nifedipina de acción prolongada: si la paciente está conciente, $10 \mathrm{mg}$ cada 30 minutos por vía oral, con una dosis máxima de 40mg (puede asociarse el sulfato de magnesio, aunque se ha informado cierta disminución de la contractilidad uterina), Clonidina: $0.15 \mathrm{mg}$ por vía intravenosa seguido por $0.75 \mathrm{mg}$ en $500 \mathrm{ml}$ de dextrosa al 5\% (5 gotas por minuto); infusión de nitroglicerina si hay edema pulmonar. El nitroprusiato de sodio se debe infundir solo en el post parto, debido al riesgo de intoxicación del feto con tiocianato. Actualmente se recomienda no utilizar hidralazina intravenosa porque parece asociarse con más efectos adversos perinatales.

Si no hay una respuesta terapéutica adecuada, las decisiones dependen de la edad gestacional: si es $>36$ semanas se debe interrumpir el embarazo. Si la edad gestacional es $<36$ semanas, deben administrarse corticoides para inducir maduración pulmonar, e interrumpirse el embarazo 48 hs más tarde.

\section{Complicaciones de la Hipertensión en el Embarazo \\ Sindrome de HELLP: Es una abreviación de los}


hallazgos principales Hemolysis, Elevated Liver enzimes, Low Platelets ${ }^{140}$, o anemia hemolítica, enzimas hepáticas elevadas, y plaquetas bajas. Junto con la eclampsia, este sindrome es la causa más frecuente de muerte materna. Puede seguir a una preeclampsia severa, o ser la primera manifestación de la enfermedad. Los tres criterios de diagnóstico son: anemia microangiopática con hiperbilirrubinemia, enzimas hepáticas y LDH elevadas, y trombocitopenia. La paciente debe ser hospitalizada, idealmente en unidad de terapia intensiva, y se recomienda interrupción del embarazo, sin importar la edad gestacional. Eclampsia: es caracterizada por convulsiones. Generalmente sigue a la preeclampsia cuando esta no es tratada adecuadamente. Se requiere internación en unidad de terapia intensiva para administrar el tratamiento adecuado ${ }^{141,142}$. El sulfato de magnesio IV ha demostrado ser efectivo para la prevención de la eclampsia y para el tratamiento de las convulsiones $^{143}$.

\section{Recomendaciones para el Seguimiento de Mujeres y Niños después de Hipertensión en el Embarazo}

Lactancia: La presión arterial de $<150 / 100 \mathrm{mmHg}$ no requiere tratamiento, y la restricción de la sal puede ser la única medida para normalizar la presión arterial. Si se requiere drogas antihipertensivas, se debe elegir las que tienen menor excreción en leche materna, tales como la metildopa, nitrendipina, captopril o enalapril ${ }^{144}$. Debe tenerse cuidado especial con los diuréticos, porque pueden inducir una disminución en la producción de leche. Los diuréticos también son excretados en la leche materna y pueden producir alteraciones electrolíticas en el neonato.

\section{Control a largo plazo de la presión arterial de la madre:}

Se debe hacer un seguimiento de la presión arterial a todas las mujeres con hipertensión en el embarazo, porque corren riesgo de presentar o desarrollar hipertensión. Es más, una serie de estudios retrospectivos ha demostrado un aumento en el riesgo cardiovascular en mujeres con preeclampsia y desnutrición intrauterina ${ }^{145-147}$.
Control de la presión arterial de los niños: Varios estudios epidemiológicos han mostrado una fuerte asociación entre el bajo peso al nacimiento y la prevalencia de la hipertensión y las enfermedades cardiovasculares en la edad adulta. Estos hallazgos apoyan el concepto que las enfermedades cardiovasculares pueden empezar en la vida intrauterina ${ }^{148}$. Por ende, se debe controlar la presión arterial de los niños hijos de madres que tuvieron hipertensión en el embarazo.

\section{Hipertensión en los Adultos Mayores}

Se conoce que la hipertensión es uno de los factores de riesgo tratables más importantes en las personas de más de 65 años de edad. La hipertensión sistólica aislada, que es muy frecuente en los adultos mayores ${ }^{150}$, conlleva un riesgo adicional porque el aumento de la presión de pulso (>65 mmHg) se asocia con mayor morbilidad y mortalidad cardiovascular. El adulto mayor es propenso a hipotensión ortostática y a seudohipertensión debido a la disminución de la distensibilidad arterial, por lo cual las mediciones de la presión arterial deben ser realizadas con el paciente en posición erguida. El monitoreo ambulatorio de la presión arterial (MAPA) de 24 horas puede

Diagnóstico: En los hipertensos adultos mayores, sobre todo en los que son resistentes al tratamiento, se debe buscar hipertensión renovascular secundaria a enfermedad ateromatosa. La ecografía doppler de arterias renales y de aorta abdominal constituye una herramienta útil para el tamizaje.

Tratamiento: En los ancianos, la presión se debe disminuir a cifras similares a las recomendadas para personas más jóvenes, es decir $<140 / 90 \mathrm{mmHg}$. Esto, sin embargo, es difícil lograr. En los ancianos la disminución de la presión arterial debe ser gradual, para asegurar una buena tolerabilidad y garantizar una buena calidad de vida. 
Varios ensayos grandes (SHEP, STOP, MRC, Sys-Eur y Sys-China ${ }^{153-157}$ han dado evidencia sólida de los beneficios de disminuir la presión arterial en pacientes mayores con hipertensión sistólica y diastólica o hipertensión sistólica aislada, demostrando disminuciones en ACV (25 a 47\%), eventos coronarios (13 a $30 \%$ ), insuficiencia cardiaca (29 a $55 \%$ ), y muerte cardiovascular (17 a 40\%) en pacientes con tratamiento activo vs placebo. El reciente ensayo $\mathrm{HYVET}^{159}$, que incluyó 3800 pacientes mayores de 80 años de edad randomizados a placebo o a tratamiento activo con indapamida y perindopril, mostró que aún en estos pacientes muy ancianos la disminución de la presión arterial se asocia con una disminución significativa en AVE fatal y no fatal (30\%), muertes por AVE (39\%), mortalidad por todas causas $(21 \%)$, muertes cardiovasculares $(23 \%)$, muertes por insuficiencia cardiaca (64\%).

Tratamiento farmacológico: Al elegir drogas antihipertensivas para los ancianos, debe tenerse en cuenta la frecuente presencia de co-morbilidades, su consecuente consumo de múltiples fármacos, y el riesgo de interacciones medicamentosas. Para evitar las caídas repentinas o excesivas de la presión arterial, debidas a farmacocinética alterada, sobre-estimación de los valores de la presión arterial, hipotensión postprandial y ortostática, disminución de la autorregulación del riego sanguíneo, etc., debe iniciarse las drogas a dosis bajas, ajustando la dosis cada 4 a 6 semanas después de evaluar los efectos colaterales. Las drogas de primera línea en los adultos mayores sin complicaciones son los diuréticos y los bloqueadores del calcio, los más usados en los ensayos clínicos, pero también hay datos favorables con otros fármacos (véase resultados del HYVET para el uso de IECA en combinación con un diurético). En los hipertensos adultos mayores con factores de riesgo asociados, complicaciones de la hipertensión o comorbilidades, debe seleccionarse la medicación según las enfermedades concomitantes. Las drogas de acción prolongada son preferibles debido a un mejor cumplimiento por parte de los pacientes (aspecto especialmente importante en los ancianos, en quienes se recomienda una posología sencilla) y un efecto antihipertensor más uniforme.

\section{REFERENCIAS:}

1. LAWES CMM, VANDER HORN S, RODGERS A, for the International Society of Hypertension. Global burden of blood pressure-related disease 2001. Lancet 2008; 371:1513-1518.

2. WHO. The World Health Report, 2002: reducing risks, promoting healthy life. Geneva: World Health Organization; 2003.

3. World Health Organization. Report of the Commission Macroeconomics and Health: investing in health for economic development. Geneva: WHO; 2001. http//whqlibdoc.who.int/publicatons/2001/9241545500x.pdf

4. Prevention of Cardiovascular Disease. Guidelines for assessment and management of Cardiovascular Risk. http://www.ish-world.com/Documents/71665 71665 _ OM S_IN T - RETIRATION.PD F

5. MANCIA G, DE BACKER G, DOMINICZAK A, CIFKOVA R, FAGARD R, GERMANO G, et al. 2007 Guidelines for the Management of Arterial Hypertension, The Task Force for the Management of Arterial Hypertension of the European Society of Hypertension (ESH) and of the European Society of Cardiology (ESC). J Hypertens 2007; 25:1105-1187.
6. BURLANDO G, SÁNCHEZ R, RAMOS F, MOGENSEN C, ZANCHETTI A, on behalf of the Latin American Experts Group. Latin American consensus on diabetes mellitus and hypertension. J Hypertens 2004; 22:2229-2241.

7. LANAS F, AVEZUM A, BAUTISTA LE, DÍAZ R, LUNA $M$, ISLAM S, et al. INTERHEART Investigators in Latin America. Risk factors for acute myocardial infarction in Latin America: the INTERHEART Latin American study. Circulation 2007; 115:1067-1074.

8. ALBALA C, VÍO F, KAIN J, UAUY R. Nutrition transition in Latin America. Nutr Rev 2001; 59:170-176.

9. SEREDAY MS, GONZÁLEZ C, GIORGINI D, DE LOREDO L, BRAGUINSKY J, COBEÑAS C, et al. Prevalence of diabetes and obesity in the central area of Argentina. Diabetes Metab 2004; 30:335-339.

10. MURRAY CJL, LOPEZ AD, editors. The global burden of disease: a comprehensive assessment of mortality, disability from diseases, injuries, risk factors in 1990, projected to 2020. Boston, Massachusetts: Harvard School of Public Health; 1996.

11.- MACMAHON S, ALDERMAN MH, LINDHOLM LH, LIU L, SÁNCHEZ RA, SEEDAT YK. Blood-pressure-related 
disease is a global health priority. Lancet 2008;1:14801482.

12. REDDY KS, YUSUF S. Emerging epidemics of cardiovascular disease in developing countries. Circulation 1998; 97:596-660.

13. JAMISON DT. Investing in health. En Disease control priorities in developing countries, $2^{\mathrm{a}}$ ed. New York: Oxford University Press; 2006. pp. 3-36.

14.- LÓPEZ-JARAMILLO P. Defining the research priorities to fight the burden of cardiovascular diseases in Latin America. J Hypertens 2008; 26:1886-1889.

15.- Actuarial Society of America and the Association of Life Insurance Medical Directors. Supplement to blood pressure study. New York: Actuarial Society of America; 1941.

16. CHOBANIAN AV, BAKRIS GL, BLACK HR, CUSHMAN WC, GREEN LA, IZZO JL, et al., and the National High Blood Pressure Education Program Coordinating Committee. The seventh report of the Joint National Committee on prevention, detection, evaluation, and treatment of high blood pressure. The JNC 7 Report. JAMA 2003; 289:2560-2572.

17. Latin American Consensus on Arterial Hypertension. J Hypertens [Spanish ed.] 2001; 6:01-110.

18. NEATON JD, WENTWORTH D. Serum cholesterol, blood pressure, cigarette smoking, and death from coronary heart disease. Overall findings and differences by age for 316,099 white men. Multiple Risk Factor Intervention Trial Research Group. Arch Intern Med 1992; 152:56-64.

19. STAMLER J, STAMLER R, NEATON JD. Blood pressure, systolic and diastolic, and cardiovascular risks. US population data. Arch Intern Med 1993; 153:598-615.

20. CUSPIDI C, MACCA G, SAMPIERI L, MICHEV I, SALERNO M, FUSI V, et al. High prevalence of cardiac and extracardiac target organ damage in refractory hypertension. J Hypertens 2001; 19:2063-2070.

21. VERDECCHIA $P$, PORCELLATI C, SCHILLACI G, BORGIONI C, CIUCCI A, BATTISTELLI $M$, et al. Ambulatory blood pressure. An independent predictor of prognosis in essential hypertension. Hypertension 1994; 24:793-801.

22. PICKERING TG, JAMES GD, BODDIE C, HARSHFIELD GA, BLANK S, LARAGH JH. How common is white coat hypertension? JAMA 1988; 259:225-228.

23. JULIUS S, MEJIA A, JONES K, KRAUSE L, SORK N, VAN DE VEN C, et al. White coat versus sustained borderline hypertension in Tecumseh, Michigan. Hypertension 1990; 16:617-623.

24. ROBLES NR, CANCHO B. Hipertensión de bata blanca. Nefrología 2002; 22 (Supl 3):72-76.

25. MANCIA G, FACCHETTI R, BOMBELLI M, GRASSI G, SEGA R. Long-term risk of mortality associated with selective and combined elevation in office, home and ambulatory blood pressure. Hypertension 2006; 47:846853.

26. OHKUBO T, KILUYA M, METOKI H, ASAYAMA K,
OBARA T, HASHIMOTO T, Et al. Prognosis of masked hypertension and white-coat hypertension detected by 24 h ambulatory blood pressure monitoring. J Am Coll Cardiol 2005; 46:508-515.

27. FAGARD RH, DEN BROSKI G, DE CORT P. Diagnostic significance of blood pressure measurement in the office, at home and during ambulatory monitoring in older patients in general population. J Hum Hypertens 2005; 19:801-807.

28. SEGA R, TROMINO G, LANZAROTTI A, CARUGO S, CESANA G, SCIAVONI R, et al. Alterations in cardiac structure in patients with isolated office, ambulatory or home hypertension. Data from the general PAMELA population. Circulation 2001; 1104:1385-1392.

29. BORDLEY J III, CONNOR CAR, HAMILTON WF, KERR WJ, WIGGERS CJ. Recommendations for human blood pressure determinations by sphygmomanometers. Circulation 1951; 4:503-509.

30. PARATI G, STERGIOU GS, ASMAR R, BILO G, DE LEEUW P, IMAI Y, KARIO K, et al., on behalf of the ESH Working Group on Blood Pressure Monitoring. European Society of Hypertension guidelines for blood pressure monitoring at home: a summary report of the Second International Consensus Conference on Home Blood Pressure Monitoring. J Hypertens 2008; 26:1505-1526.

31. PALATINI P. Ambulatory blood pressure monitoring in clinical practice: is being superior good enough? J Hypertens 2008; 26:1300-1302.

32. WHITE WB. Ambulatory blood pressure monitoring in clinical practice. N Engl J Med 2003; 348:2377-2378.

33. Asociación Latinoamericana de Diabetes. Guías ALAD 2002 para el Diagnostico y Manejo de la Diabetes Mellitus tipo 2 con Medicina basada en Evidencia. http://www. fenadiabetes.org.ve/docs/guia.pdf.

34. FORD ES, GILES WH. A comparison of the prevalence of the metabolic syndrome using two proposed definitions. Diabetes Care 2003; 26:575-581.

35. NINOMIYA JK, L'ITALIEN G, CRIQUI MH, WHYTE JL, GAMST A, CHEN RS. Association of the metabolic syndrome with history of myocardial infarction and stroke in the third national health and nutrition examination survey. Circulation 2004; 109:42-46.

36. LAKKA HM, LAAKSONEN DE, LAKKA TA, NISKANEN LK, KUMPUSALO E, TUOMILEHTO J, SALONEN JT. The metabolic syndrome and total and cardiovascular disease mortality in middle-aged men. JAMA 2002; 288:2709-2716.

37. LÓPEZ-JARAMILLO P, RUEDA-CLAUSEN C, SILVA FA. The utility of different definitions of metabolic syndrome in Andean population. Int J Cardiol 2007; 116:421-422.

38. GARCÍA RG, CIFUENTES AE, CABALLERO RS, SÁNCHEZ L, LÓPEZ-JARAMILLO P. A Proposal for an appropriate central obesity diagnosis in Latin American population. Int J Cardiol 2005; 110:263-264.

39. PÉREZ M, CASAS JP, CUBILLOS LA, SERRANO NC, SILVA FA, MORILLO CA, LÓPEZ- JARAMILLO P. Using 
waist circumference as screening tool to identify colombian subjects at cardiovascular risk. Eur J Cardiovasc Prev Rehabil 2003; 10:328-335.

40. The DREAM trial investigators. Effect of ramipril on the incidence of diabetes. N Engl J Med 2006; 355:15511562.

41. National Institutes of Health. Third Report of the National Cholesterol Education Program Expert Panel on detection, evaluation, and treatment of high blood cholesterol in adults (Adult Treatment Panel III). Executive summary. Bethesda, MD: National Institutes

42. ALEXANDER CM, LANDSMAN PB, TEUTSCH SM, HAFFNER SM. Third National Health and Nutrition Examination Survey (NHANES III); National Cholesterol Education Program (NCEP). NCEP-defined metabolic syndrome, diabetes, and prevalence of coronary heart disease among NHANES III participants age 50 years and older. Diabetes 2003; 52:1210-1214.

43. COUTINHO M, GERSTEIN HC, WANG Y, YUSUF S. The relationship between glucose and incident cardiovascular events. A metaregression analysis of published data from 20 studies of 95,783 individuals followed for 12.4 years. Diabetes Care 1999; 22:233-240.

44. LEVITAN EB, SONG Y, FORD ES, LIU S. Is nondiabetic hyperglycemia a risk factor for cardiovascular disease? A meta-analysis of prospective studies. Arch Intern Med 2004; 25:2147-2155.

45. GERSTEINHC, PAIS P, POGUE J, YUSUF S. Relationship of glucose and insulin levels to the risk of myocardial infarction: a case control study. J Am Coll Cardiol 1999; 33:612-619.

46. Asia Pacific Cohort Studies Collaboration. Blood glucose and risk of cardiovascular disease in the Asian Pacific region. Diabetes Care 2004; 27:2836-2842.

47. LÓPEZ-JARAMILLO P, PRADILLA LP, CASTILLO V, LAHERA V. Socio-economical pathology as determinant of regional differences in the prevalence of metabolic syndrome and pregnancy-induced hypertension. Rev Esp Cardiol 2007; 60:168-178.

48. ADA Clinical Practice Recommendations. Diagnosis and classification of diabetes mellitus. Diabetes Care 2004; 27(Suppl 1):S5-S10.

49. SAWICKI PT, MUÜLHAUSER I, DIDJURGEIT U, REIMANN M, BENDER R, BERGER M. Mortality and morbidity in treated hypertensive type 2 diabetic patients with micro-proteinuria. Diabetic Med 1995; 12:893-898.

50. SOWERS JR, EPSTEIN M, FROHLICH ED. Diabetes, hypertension, and cardiovascular disease: an update. Hypertension 2001; 37:1053-1059.

51. JAMES WPT, JACKSON-LEACH R, MHURDU CN, KALAMARA E, SHAYEGHI M, RIGBY N, et al. Overweight and obesity. En: Ezzati M, Lopez AD, Rodgers A, Murray CJL, editores. Comparative quantification of health risks: global and regional burden of disease attributable to selected major risk factors. Geneva: WHO; 2003.

52. ERIKSSON H, WELIM L, WILHELMSEN L, LARSSON
B, OHLSON LO, Svardsudd K, Tibblin G. Metabolic disturbances in hypertension: results from the population study: 'men born in 1913'. J Intern Med 1992; 232:389395.

53. NELSONRG, BENNETTPH, BECK GJ, TAN M, KNOWLER WC, MITCH WE, et al. Development and progression of renal disease in Pima Indians with noninsulin-dependent diabetes mellitus. N Engl J Med 1996; 335:1636-1642.

54. BAKRIS GL, WILLIAMS M, DWORKIN L, ELLIOTT WJ, EPSTEIN M, TOTO $R$, et al. Preserving renal function in adults with hypertension and diabetes: a consensus approach. Am J Kidney Dis 2000; 36:646-661.

55. DEFRONZO RA. Diabetic nephropathy: etiologic and therapeutic considerations. Diabetes Rev 1995; 3:510564.

56. LEWIS EJ, HUNSICKER LG, CLARKE WR, BERL T, POHL MA, LEWIS JB, et al. Renoprotective effect of the angiotensin-receptor antagonist irbesartan in patients with nephropathy due to type 2 diabetes. N Engl J Med 2001; 345:851-860.

57. BRENNER BM, COOPER ME, DE ZEEUW D, KEANE WF, MITCH WE, PARVING HH, et al., for the RENAAL Study Investigators. Effects of losartan on renal and cardiovascular outcomes in patients with type 2 diabetes and nephropathy. N Engl J Med 2001; 345:861-869.

58. MOGENSEN CE, VIBERTI G, HALIMI S, RITZ E, RUILOPE L, JERMENDY G, et al., Preterax in Albuminuria Regression (PREMIER) Study Group. Effect of low-dose perindopril/indapamide on albuminuria in diabetes: preterax in albuminuria regression: PREMIER. Hypertension 2003; 41:1063-1071.

59. The ONTARGET Investigators. Telmisartan, ramipril or both in patients with high risk for vascular events. N Engl J Med 2008; 358:1547-1559.

60. PEDRINI MT, LEVEY AS, LAU J, CHALMERS TC, WANG PH. The effect of dietary protein restriction on the progression of diabetic renal diseases: a metaanalysis. Ann Intern Med 1996; 124:627-632.

61. LEE WL, CHEUNG AM, CAPE D, ZINMAN B. Impact of diabetes on coronary artery disease in women and men: a meta analysis of prospective studies. Diabetes Care 2000; 23:962-968.

62. BOOTH GL, KAPRAL MK, FUNG K, TU JV. Relation between age and cardiovascular disease in men and women with diabetes compared with nondiabetic people: a population-based retrospective cohort study. Lancet 2006; 368:29-36.

63. COLWELL JA. Aspirin therapy in diabetes [technical review]. Diabetes Care 1997; 20:1767-1771.

64. HANSSON L, ZANCHETTI A, CARRUTHERS SG, DAHLOF B, ELMFELDT D, JULIUS $S$, et al. Effects of intensive blood-pressure lowering and low-dose aspirin in patients with hypertension: principal results of the Hypertension Optimal Treatment (HOT) randomised trial. Lancet 1998; 351:1755-1762.

65. BONOW RO, MITCH WE, NESTO RW, O'GARA PT, 
BECKER RC, CLARK LT, et al. Prevention conference VI. Diabetes and Cardiovascular disease. Writing Group $\mathrm{V}$ : management of cardiovascular-renal complications. Circulation 2002; 105:e159-e164.

66. MCDONAGH TA, MORRISON CE, LAWRENCE A, FORD I, TUNSTALL-PEDOE H, MCMURRAY JJ, DARGIE HJ. Symptomatic and asymptomatic left ventricular systolic dysfunction in an urban population. Lancet 1997; 350:829_ 833.

67. KANNEL WB, HJORTLAND M, CASTELLI WP. Role of diabetes in congestive HF: the Framingham study. Am J Cardiol 1974; 34:29-34.

68. IRIBARREN C, KARTER AJ, GO AS, FERRARAA, LIU JY, SIDNEY S, SELBY JV. Glycaemic control and HF among adult patients with diabetes. Circulation 2001; 103:26682673.

69. Bell DS. The frequent, forgotten and often fatal complication of diabetes. Diabetes Care 2003; 26:2433-2441.

70. MARANTZ PR, TOBIN JN, WASSERTHEIL-SMOLLER $\mathrm{S}$, STEINGART RM, WEXLER JP, BUDNER $\mathrm{N}$, et al. The relationship between left ventricular function and congestive HF diagnosed by clinical criteria. Circulation 1988; 77:607-612.

71. KASRAPANAYIOTIDES T, PIECHOWSKI-JOZWIAK B, VAN MELLE G, BOGOUSSLAVSKY J, DEVUYST G. Stroke patterns, etiology and prognosis in patients with diabetes mellitus. Neurology 2004; 62:1558-1562.

72. MEGHERBI SE, MILAN C, MINIER D, COUVREUR G, OSSEBY GV, TILLING K, et al. Association between diabetes and stroke subtype on survival and functional outcome 3 months after stroke: data from the European BIOMED Stroke Protect. Stroke 2003; 34:688-694.

73. Consenso de Hipertensión Arterial. Rev Argent Cardiol 2007; 75(Suppl 3):1-43.

74. The Diabetes Controland Complications Trial/Epidemiology of Diabetes Interventions and Complications (DCCT/EDIC) Study Research Group. intensive diabetes treatment and cardiovascular disease in patients with type 1 diabetes. $\mathrm{N}$ Engl J Med 2005; 353:2643-2653.

75. UK Prospective Diabetes Study (UKPDS) Group. Intensive blood glucose control with sulphonylureas or insulin compared with conventional treatment and risk of complications in patients with type diabetes (UKPDS). Lancet 1998; 352:837-853.

76. The Action to Control Cardiovascular Risk in Diabetes Study Group. Effects of intensive glucose lowering in type 2 diabetes. N Engl J Med 2008; 358:2545-2559.

77. The ADVANCE Collaborative Group. Intensive blood glucose control and vascular outcomes in patients with type 2 diabetes. N Engl J Med 2008; 358:2560-2572.

78. MELLBIN LG, MALMBERG K, NORHAMMAR A, WEDEL $H$, RYDEN L, for the DIGAMI 2 Investigators. The impact of glucose lowering treatment on long-term prognosis in patients with type 2 diabetes andmyocardial infarction: a report from the DIGAMI 2 trial. Eur Heart J 2008; 29:166176.
79. GARCÍA RG, LÓPEZ-JARAMILLO P. Cardiovascular prevention in high-risk patients with type 2 diabetes mellitus: when to start it? Eur Heart J 2008; 29:20582059.

80. GRESS TW, NIETO FJ, SHAHAR E, WOFFORD MR, BRANCATI FL. Hypertension and antihypertensive therapy as risk factors for type 2 diabetes mellitus. Atherosclerosis Risk in Communities study. N Engl J Med 2000; 342:905912.

81. CARLSSON PO, BERNE C, JANSSON L. Angiotensin II and the endocrine pancreas: effects on islet blood flow and insulin secretion in rats. Diabetologia 1998; 41:127-133.

82. FERRANNINI E, SEGHIERI G, MUSCELLI E. Insulin and the renin-angiotensin-aldosterone system: influence of ACE inhibition. J Cardiovasc Pharmacol 1994; 24 (Suppl 3):S61-S69.

83. VELLOSO LA, FOLLI F, SUN XJ, WHITE MF, SAAD MJ, KAHN CR. Cross-talk between the insulin and angiotensin signaling systems. Proc Natl Acad Sci USA 1996; 93:12490-12495.

84. ESLER M. Differentiation in the effects of the angiotensin II receptor blocker class on autonomic function. J Hypertens Suppl 2002; 20:S13-S19.

85. TORLONE E, RAMBOTTI AM, PERRIELLO G, BOTTA G, SANTEUSANIO F, BRUNETTI P, BOLLI GB. ACEinhibition increases hepatic and extrahepatic sensitivity to insulin in patients with type 2 (noninsulin-dependent) diabetes mellitus and arterial hypertension. Diabetologia 1991; 34:119-125.

86. GILLESPIE EL, WHITE CM, KARDAS M, LINDBERG M, COLEMAN CI. The impact of ACE inhibitors or angiotensin II type 1 receptor blockers on the development of newonset type 2 diabetes. Diabetes Care 2005; 28:22612266.

87. ELLIOTT WJ, MEIER PM. Incident diabetes in clinical trials of antihypertensive drugs: a network meta-analysis. Lancet 2007; 369:201-207.

88 LÓPEZ-JARAMILLO P, PRADILLA LP, LAHERA V, SILVA F, RUEDA-CLAUSEN C, MÁRQUEZ G. A randomized, double blind, cross-over, placebo-controlled clinical trial to assess the effects of candesartan on the insulin sensitivity on non diabetic, non hypertenive subjects with dysglycemia and abdominal obesity. ARAMIA. Trials 2006; 7:28-39.

89. LÓPEZ-JARAMILLO P, GARCÍA G, CAMACHO PA, HERRERA E, CASTILLO V. Interrelationship between body mass index, C-reactive protein and blood pressure in a Hispanic pediatric population. Am J Hypertens 2008; 21:527-532.

90. LÓPEZ-JARAMILLO P, SILVA SY, RODRÍGUEZ SALAMANCAN, DURÁNA, MOSQUERAW, CASTILLO V. Are nutrition-induced epigenetic changes the link between socioeconomic pathology and cardiovascular diseases? Am J Therapeutic 2008; 15:362-372.

91. RUEDA-CLAUSEN C, SILVA F, LÓPEZ-JARAMILLO P. Epidemic of obesity and overweigh in Latin America and the Caribbean. Int J Cardiol 2008; 125:111-112. 
92. RUEDA-CLAUSEN CF, LAHERA V, CALDERÓN J, BOLIVAR IC, CASTILLO VR, GUTIÉRREZ M, et al. The presence of abdominal obesity is associated with changes in vascular function independently of other cardiovascular risk factors. Int J Cardiol 2008 [published online 15 October 2008]. http://www.internationaljournalofcardiology.com/ article/S0167-5273(08)01012-7

93. MANCIA G, OMBONI S, PARATI G, CLEMENT DL, HALEY WE, RAHMAN SN, et al. Twenty-four hour ambulatory blood pressure in the Hypertension Optimal Treatment (HOT) study. J Hypertens 2001; 19:1755-1763.

94. BECKETT NS, PETERS R, FLETCHER AE, STAESSEN JA, LIU K, DUMITRASCU D, et al., for the HYVET Study Group. Treatment of hypertension in patients 80 years of age or older. N Engl J Med 2008; 358:1887-1898.

95. MANCIA G, OMBONI S, RAVOGLI A, PARATI G, ZANCHETTI A. Ambulatory blood pressure monitoring in the evaluation of antihypertensive treatment: additional information from a large data base. Blood Press 1995; 4:148-156.

96. MANCIA G, PARATI G, BILO G, MARONATI A, OMBONI S, HENNIG M, et al. Assessment of long-term antihypertensive treatment by clinic an ambulatory blood pressure. Data from the ELSA Study. J Hypertens 2007; 25:1087-1094.

97. FAGARD RH, STAESSEN JA, THIJS L. Relationships between changes in left ventricular mass and in clinic and ambulatory blood pressure in response to antihypertensive therapy. J Hypertens 1997; 15:1493-1502.

99. GOSSE P, SHERIDAN DJ, ZANNAD F, DUBOURG O, GUERET P, KARPOV Y, et al., on behalf of the LIVE investigators. Regression of left ventricular hypertrophy in hypertensive patients treated with indapamide SR $1.5 \mathrm{mg}$ versus enalapril 20mg: the LIVE study. J Hypertens 2000; 18:1465-1475.

100.DANDONA P, GHANIM H, BROOKS DP. Antioxidant activity of carvedilol in cardiovascular disease. J Hypertens 2007; 25:731-741.

101.POIRIER L, CLEROUX J, NADEAU A, LACOURCIERE Y. Effects of nebivolol and atenolol on insulin sensitivity and haemodynamics in hypertensive patients. J Hypertens 2001; 19:1429-1435.

102. KRUM H, GILBERT RE. Novel therapies blocking the renin-angiotensin-aldosterone system in the management of hypertension and related disorders. J Hypertens 2007; 25:25-35.

103.NEUTEL JM, SICA DA, FRANKLIN SS. Hypertension control: still not there - how to select the right add-on therapy to reach goal blood pressures. J Clin Hypertens 2007; 9:889-896.

104.WAEBER B, BURNIER M, BRUNNER HR. Compliance with antihypertensive therapy. Clin Exp Hypertens 1999; 21:973-985.

105. National High Blood Pressure Education Program Working Group on High Blood Pressure in Children and Adolescents. The Fourth Report on the diagnosis, evaluation, and treatment of high blood pressure in children and adolescents. Pediatrics 2004; 111:555-576.

106. FIXLER DE, LAIRD WP, FITZGERALD V, STEAD S, ADAMS R. Hypertension screening in schools: results of the Dallas study. Pediatrics 1979; 63:32-36.

107.SOROF JM, LAI D, TURNER J, POFFENBARGER T, PORTMAN RJ. Overweight, ethnicity and the prevalence of hypertension in school-aged children. Pediatrics 2004; 113:475-482.

108. HANSEN ML, GUNN PW, KAELBER DC. Underdiagnosis of hypertension in children and adolescents. J Am Med Assoc 2007; 298:874-879.

109. HAVLIK RJ, GARRISON RJ, FEINLEIB M, KANNEL WB, CASTELLI WP, MCNAMARA PM. Blood pressure aggregation in families. Am J Epidemiol 1979; 110:304312.

110. LAUER RM, CLARKE WR. Childhood risk factors for high adult blood pressure: the Muscatine study. Pediatrics 1989; 84:633-641.

111. BAO W, THREEFOOT SA, SRINIVASAN SR, BERENSON GS. Essential hypertension predicted by tracking of elevated blood pressure from childhood to adulthood: the Bogalusa Heart Study. Am J Hypertens 1995; 8:657-665.

112. HORNSBY JL, MONGAN PF, TAYLOR AT, TREIBER FA. White coat hypertension in children. J Fam Pract 1991; 33:617-623.

113. SOROF JM, PORTMAN RJ. White coat hypertension in children with elevated casual blood pressure. J Pediatr. 2000;137:493-497.

114. WÜHL E, WITTE K, SOERGEL M, MEHLS O, SCHAEFER $F$, on behalf of the German Working Group on Pediatric Hypertension. Distribution of 24-h ambulatory blood pressure in children: normalized reference values and role of body dimensions. J Hypertens 2002; 20:1995-2007.

115. BRANDAO AP, BRANDAO AA, ARAUJO EM, OLIVEIRA RC. Familial aggregation of arterial blood pressure and possible genetic influence. Hypertension 1992; 19 (Suppl 2):214-217.

116. LURBE E, TORRO I, AGUILAR F, ALVÁREZ J, ALCÓN J, PASCUAL JM, REDÓN J. ADDED impact of obesity and insulin resistance in nocturnal blood pressure elevation in children and adolescents. Hypertension 2008; 51:635641.

117. HE FJ, MARRERO NM, MAC GREGOR GA. Salt intake is related to soft drink consumption in children and adolescents: a link to obesity? Hypertension 2008; 51:629-634.

118.DOLEA C, ABOUZAHR C. GLOBAL burden of hypertensive disorders of pregnancy in the year 2000. Evidence and information for policy (EIP). Geneva: World Health Organization; 2003. http://www.who.int/ healthinfo/ statistics/bod_hypertensivedisordersofpregnancy.pdf.

119. Report of the National High Blood Pressure Education Program Working Group on High Blood Pressure in Pregnancy. Am J Obstet Gynecol 2000; 183:S1-S22.

120.RED-HORSE K, ZHOU Y,GENBACEV O, PRAKOBPHOL 
A, FOULK R,MCMASTER M, FI SJ. Trophoblast differentiation during embryo implantation and formation of the maternal-fetal interface. J Clin Invest 2004; 114:744754.

121.DAVISON JM, HOMUTH V, JEYABALAN A, CONRAD KP, ANANTH KARUMANCHI S, QUAGGIN $S$, et al. New aspects in the pathophysiology of preeclampsia. J Am Soc Nephrol 2004; 15:2440-2448.

122. SIBAI BM, EL-NAZER A, GONZALEZ-RUIZ A. Severe preeclampsia-eclampsia in young primigravid women: subsequent pregnancy outcome and remote prognosis. Am J Obstet Gynecol 1986; 155:1011-1016.

123.LÓPEZ-JARAMILLO P, GARCIA R, LÓPEZ M. Preventing pregnancy induced hypertension: are there regional differences for this global problem? J Hypertens 2005; 23:1121-1129.

124. GARCÍA R, CELEDÓN J, SIERRA J, ALARCÓN M, LUENGAS C, SILVA F, LÓPEZ- JARAMILLO P. RAISED C-reactive protein and impaired flow mediated vasodilation precede the development of preeclampsia. Am J Hypertens 2007; 20:98-103.

125. TERÁN E, ESCUDERO C, MOYA W, FLORES $M$, VALLANCE P, LÓPEZ-JARAMILLO P. Elevated C-reactive protein and pro-inflammatory cytokines in Andean women with preeclampsia. Int J Gynaecol Obstet 2001; 75:243249.

126. HERRERA JA, PARRA B, HERRERA E, BOTERO JE, ARCE RM, CONTRERAS A, LÓPEZ- JARAMILLO P. Periodontal disease severity is related to high levels of Creactive protein in preeclampsia. J Hypertens 2007; 25:1459-1464

127.SIERRA-LAGUADO J, GARCÍA RG, CELEDÓN J, ARENAS-MANTILLA M, PRADILLA LP, CAMACHO PA, LÓPEZ-JARAMILLO P. Determination of insulin resistance using the homeostatic model assessment (HOMA) and its relation with the risk of developing pregnancy-induced hypertension. Am J Hypertens 2007; 20:437-442.

128. KNIGHT M, DULEY L, HENDERSONS-SMART DJ, KING JF. Antiplatelet agents and preeclampsia (Cochrane review). The Cochrane Library, issue 1, Oxford, update software, 2000.

129. BELIZAN JM, VILLAR J. The relationship between calcium intake and edema-, proteinuria-, and hypertension-getosis: an hypothesis. Am J Clin Nutr 1980; 33:2202-2210.

130.LÓPEZ-JARAMILLO P, NARVÁEZ M, WEIGEL M, YÉPEZ R. Calcium supplementation reduces the risk of pregnancy induced hypertension in an Andean population. Br J Obstet Gynecol 1989; 96:648-655.

131. VILLAR J, ABDEL-ALEEM H, MERIALDI M, MATHAI M, ALI MM, ZAVALETA N, et al. World Health Organization Calcium Supplementation for the Prevention of Preeclampsia Trial Group: World Health Organization randomized trial of calcium supplementation among low calcium intake pregnant women. Am J Obstet Gynecol 2006; 194:639-649.

132. HOFMEYR GJ, ATALLAH AN, DULEY L. Calcium supplementation during pregnancy for preventing hypertensive disorders and related problems. Cochrane Database Syst Rev 2006; 3:CD001059.

133. HERRERA JA, CHAUDHURI G, LÓPEZ-JARAMILLO $P$. Is infection a major risk factor for preeclampsia? MedHyotheses 2001; 57:393-397.

134.DEKKER GA, DE VRIES JIP, DOELITZSCH PM, HUIJGENS PC, VON BLOMBERG BME, JAKOBS C, VAN GEIJN HP. Underlying disorders associated with severe early-onset preeclampsia. Am J Obstet Gynecol 1995; 173:1042-1048.

135.KUPFERMINC MJ, ELDOR A, STEINMAN N, MANY A, BAR-AM A, JAFFAA, et al. Increased frequency of genetic thrombophilia in women with complications of pregnancy. N Engl J Med 1999; 340:9-13.

136.PODYMOW T, AUGUST P. Update on the use of antihypertensive drugs in pregnancy. Hypertension 2008; 51:960-969.

137. CUNNIFF C, JONES KL, PHILLIPSON J, BENIRSCHKE K, SHORT S, WUJEK J. Oligohydramnios sequence and renal tubular malformation associated with maternal enalapril use. Am J Obstet Gynecol 1990; 162:187-189.

138. HANSSENS M, KEIRSE MJ, VANKELECOM F, VAN ASSCHE FA. Fetal and neonatal effects of treatment with angiotensin-converting enzyme inhibitors in pregnancy. Obstet Gynecol 1991; 78:128-135.

139.LYDAKIS C, LIP GY, BEEVERS M, BEEVERS DG. Atenolol and fetal growth in pregnancies complicated by bypertension. Am J Hypertens 1999; 12:541-547.

140.STONE JH. HELLP syndrome: hemolysis, elevated liver enzymes, and low platelets. JAMA 1998; 280:559-562.

141. Aagaard-Tillery KM, Belfort MA. Eclampsia: morbidity, mortality, and management. Clin Obstet Gynecol 2005; 48:12-23.

142. SIBAI BM. Diagnosis, prevention, and management of eclampsia. Obstet Gynecol 2005; 105:402-410.

143.Magpie Trial Collaborative Group. Do women with preeclampsia, and their babies, benefit from magnesium sulphate? The Magpie Trial: a randomised placebocontrolled trial. Lancet 2002; 359:1877-1890.

144.BEARDMORE KS, MORRIS JM, GALLERY ED. Excretion of antihypertensive medication into human breast milk: a systematic review. Hypertens Pregnancy 2002; 21:85-95.

145. ARNADOTTIR GA, GEIRSSON RT, ARNGRIMSSON R, JONSDOTTIR LS, OLAFSSON O. Cardiovascular death in women who had hypertension in pregnancy: a casecontrol study. Br J Obstet Gynecol 2005; 112:286-292.

146. IRGENS HU, REISAETER L, IRGENS LM, LIE RT. Long term mortality of mothers and fathers after preeclampsia: population based cohort study. BMJ 2001; 323:12131217.

147.SMITH GC, PELL JP, WALSH D. Pregnancy complications and maternal risk of ischaemic heart disease: a retrospective cohort study of 129,290 births. Lancet 2001 ; 357:2002-2006.

148.BARKER DJ, BAGBY SP, HANSON MA. Mechanisms 
of disease: in utero programming in the pathogenesis of hypertension. Nat Clin Pract Nephrol 2006; 2:700-707.

149.FRANKLIN SS, GUSTIN IVM, WONG ND, LARSON MG, WEBER MA, KANNEL WB, LEVY D. Hemodynamic patterns of age-related changes in blood pressure. The Framingham Heart Study. Circulation 1997; 96:308-315.

150.BENETOS A, SAFAR M, RUDNICHI A, SMULYAN $H$, RICHARD JL, DUCIMETIE' RE P, GUIZE J. Pulse pressure: a predictor of long-term cardiovascular mortality in a French male population. Hypertension 1997; 30:14101415.

151.SILAGY CA, MCNEIL JJ, FARISH S, MCGRATH BP. Comparison of repeated measurement of ambulatory and clinic blood pressure readings in isolated systolic hypertension. Clin Exp Hypertens 1993; 15:895-909.

152.WIINBERG N, HØEGHOLMA, CHRISTENSEN HR, BANG LE, MIKKELSEN KL, NIELSEN PE, et al. 24-h ambulatory blood pressure in 352 normal Danish subjects, related to age and gender. Am J Hypertens 1995; 8:978-986.

153.SHEP Collaborative Research Group. Prevention of stroke by antihypertensive drug treatment in older persons with isolated systolic hypertension: final results of the Systolic Hypertension in the Elderly Program (SHEP). JAMA 1991; 265:3255-3264.

154.DAHLOF B, LINDHOLM LH, HANSSON L, SCHERSTEN $B$, EKBOM T, WESTER PO. Morbidity and mortality in the Swedish Trial in Old Patients with Hypertension (STOPHypertension). Lancet 1991; 338:1281-1285.

155. Medical Research Council trial of treatment of hypertension in older adults: principal, results. MRC Working Party. BMJ 1992; 304:405-412.

156. STAESSEN JA, FAGARD R, THIJS L, CELIS H, ARABIDZE GG, BIRKENHA"GER WH, et al. Randomised doubleblind comparison of placebo and active treatment for older patients with isolated systolic hypertension. The Systolic Hypertension in Europe (Syst-Eur) Trial Investigators. Lancet 1997; 350:757-764.

157.LIU L, WANG JG, GONG L, LIU G, STAESSEN JA. Comparison of active treatment and placebo in older Chinese patients with isolated systolic hypertension. Systolic Hypertension in China (Syst-China) Collaborative Group. J Hypertens 1998; 16:1823-1829. 